\title{
Multiple singular integrals and maximal operators related to homogeneous mappings
}

Feng Liu ${ }^{1 *}$, Daiqing Zhang ${ }^{2}$ and Jing Zhang ${ }^{3}$

"Correspondence:

liufeng860314@163.com

${ }^{1}$ College of Mathematics and

Systems Science, Shandong

University of Science and

Technology, Qingdao, Shandong

266590, China

Full list of author information is

available at the end of the article

\begin{abstract}
In this paper, we present the $L^{p}$ mapping properties of multiple singular integrals related to homogeneous mappings with rough kernels given by the radial function $h \in \Delta_{\gamma}$ (or $h \in U_{\gamma}$ ) for some $\gamma>1$ (or $\gamma \geq 1$ ) and the sphere function $\Omega \in L\left(\log ^{+} L\right)^{2}\left(S^{m-1} \times S^{n-1}\right)\left(\operatorname{or} \Omega \in L\left(\log ^{+} L\right)^{2 / \gamma^{\prime}}\left(S^{m-1} \times S^{n-1}\right)\right)$. In addition, the $L^{p}$ bounds for the related maximal operators are also given. Our main results extend and improve some known ones.
\end{abstract}

MSC: 42B20; 42B25

Keywords: multiple singular integrals; rough kernels; maximal operators; homogeneous mappings

\section{Introduction}

Let $\mathbb{R}^{\Lambda}(\Lambda=m$ or $n), \Lambda \geq 2$, be the $\Lambda$-dimensional Euclidean space, and $S^{\Lambda-1}$ denote the unit sphere in $\mathbb{R}^{\Lambda}$ equipped with the induced Lebesgue measure $d \sigma_{\Lambda}$. For any $x \in \mathbb{R}^{\Lambda}$, we also let $x^{\prime}=x /|x|$. Let $\Omega \in L^{1}\left(\mathbb{R}^{m} \times \mathbb{R}^{n}\right)$ satisfy

$$
\int_{S^{m-1}} \Omega\left(u^{\prime}, \cdot\right) d \sigma_{m}\left(u^{\prime}\right)=\int_{S^{n-1}} \Omega\left(\cdot, v^{\prime}\right) d \sigma_{n}\left(v^{\prime}\right)=0 .
$$

For $M, N \geq 2$ and two suitable mappings: $\Gamma: \mathbb{R}^{m} \rightarrow \mathbb{R}^{M}$ and $\Upsilon: \mathbb{R}^{n} \rightarrow \mathbb{R}^{N}$, define the multiple singular integral operators $T_{h, \Omega, \Gamma, \Upsilon}$ along the surfaces $S_{\Gamma, \Upsilon}=\{(\Gamma(u), \Upsilon(v)):(u, v) \in$ $\left.\mathbb{R}^{m} \times \mathbb{R}^{n}\right\}$ by

$$
T_{h, \Omega, \Gamma, \Upsilon}(f)(x, y):=\text { p.v. } \iint_{\mathbb{R}^{m} \times \mathbb{R}^{n}} f(x-\Gamma(u), y-\Upsilon(v)) K_{h, \Omega}(u, v) d u d v,
$$

where $K_{h, \Omega}(u, v)=\Omega\left(u^{\prime}, v^{\prime}\right) h(|u|,|v|)|u|^{-m}|v|^{-n}$ and $h \in \Delta_{1}\left(\mathbb{R}_{+} \times \mathbb{R}_{+}\right)$. Here $\mathbb{R}_{+}=(0, \infty)$, and $\Delta_{\gamma}\left(\mathbb{R}_{+} \times \mathbb{R}_{+}\right)(\gamma \geq 1)$ is the set of all measurable functions $h(r, s)$ on $\mathbb{R}_{+} \times \mathbb{R}_{+}$satisfying the condition

$$
\|h\|_{\Delta_{\gamma}\left(\mathbb{R}_{+} \times \mathbb{R}_{+}\right)}:=\sup _{k, j \in \mathbb{Z}}\left(\int_{2^{j}}^{2^{j+1}} \int_{2^{k}}^{2^{k+1}}|h(r, s)|^{\gamma} \frac{d r d s}{r s}\right)^{1 / \gamma}<\infty .
$$

For convenience, we denote $\Delta_{\gamma}\left(\mathbb{R}_{+} \times \mathbb{R}_{+}\right)$by $\Delta_{\gamma}$ for $\gamma \geq 1$. Obviously, $\Delta_{\gamma_{1}} \subsetneq \Delta_{\gamma_{2}}$ for $\gamma_{1}>$ $\gamma_{2}>0$.

(c) 2016 Liu et al. This article is distributed under the terms of the Creative Commons Attribution 4.0 International License (http://creativecommons.org/licenses/by/4.0/), which permits unrestricted use, distribution, and reproduction in any medium, provided you give appropriate credit to the original author(s) and the source, provide a link to the Creative Commons license, and indicate if changes were made. 
For simplicity, we denote $T_{h, \Omega, \Gamma, \Upsilon}=T_{\Omega, \Gamma, \Upsilon}$ if $h \equiv 1$ and $T_{\Omega, \Gamma, \Upsilon}=T_{\Omega}$ if $M=m, N=n$, $\Gamma(u)=u$, and $\Upsilon(v)=v$. The operator $T_{\Omega}$ is the classic multiple singular integral operator, which was first introduced by Fefferman and Stein (see $[1,2])$ and has been studied extensively by many authors (see [3-6], etc.). In particular, Duoandikoetxea [4] proved that $T_{\Omega}$ is bounded on $L^{p}\left(\mathbb{R}^{m} \times \mathbb{R}^{n}\right)$ for $1<p<\infty$, provided that $\Omega \in L^{q}\left(S^{m-1} \times S^{n-1}\right)$ with $q>1$. Later on, Chen [7] improved the result of [4] to the case $\Omega \in L\left(\log ^{+} L\right)^{2}\left(S^{m-1} \times S^{n-1}\right)$. Subsequently, Ying and Chen [8] (resp., Al-Salman et al. [3]) extended the result of [7] to the multiple singular integrals along polynomial curves (resp., associated with polynomial mappings). We also refer the readers to [9-11], among others. Recall that $\Omega \in$ $L\left(\log ^{+} L\right)^{\alpha}\left(S^{m-1} \times S^{n-1}\right)$ for $\alpha>0$ is the set of all functions $\Omega$ on $S^{m-1} \times S^{n-1}$ satisfying

$$
\|\Omega\|_{L\left(\log ^{+} L\right)^{\alpha}\left(S^{m-1} \times S^{n-1}\right)}:=\iint_{S^{m-1} \times S^{n-1}}|\Omega(u, v)| \log ^{\alpha}(2+|\Omega(u, v)|) d \sigma_{m}(u) d \sigma_{n}(v)<\infty .
$$

Note that, for any $q>1$ and $\beta>\alpha>0$,

$$
L^{q}\left(S^{m-1} \times S^{n-1}\right) \subsetneq L\left(\log ^{+} L\right)^{\beta}\left(S^{m-1} \times S^{n-1}\right) \subsetneq L\left(\log ^{+} L\right)^{\alpha}\left(S^{m-1} \times S^{n-1}\right) .
$$

The aim of this paper is to investigate the $L^{p}$ bounds for multiple singular integral operators associated with homogeneous mappings and the related maximal operators. For $l \in \mathbb{N} \backslash\{0\}$ and $d=\left(d_{1}, \ldots, d_{l}\right) \in \mathbb{R}^{l}$, define the family of dilations $\left\{\delta_{t}\right\}_{t>0}$ on $\mathbb{R}^{l}$ by

$$
\delta_{t}\left(x_{1}, \ldots, x_{l}\right)=\left(t^{d_{1}} x_{1}, \ldots, t^{d_{l}} x_{l}\right) .
$$

We say that a mapping $\Phi: \mathbb{R}^{n} \rightarrow \mathbb{R}^{l}$ is homogeneous of degree $d$ if

$$
\Phi(t x)=\delta_{t}(\Phi(x))
$$

for all $x \in \mathbb{R}^{n}$ and $t>0$. When $\Gamma, \Upsilon$ are two homogeneous mappings, Al-Qassem and Ali [12] proved that $T_{\Omega, \Gamma, \Upsilon}$ is bounded on $L^{p}\left(\mathbb{R}^{M} \times \mathbb{R}^{N}\right)$ for $1<p<\infty$ if $\Omega \in B_{q}^{(0,1)}\left(S^{m-1} \times\right.$ $\left.S^{n-1}\right)$ for some $q>1$. We note that the question with regard to the relationship between $B_{q}^{(0, \alpha-1)}\left(S^{m-1} \times S^{n-1}\right)$ with $q>1$ and $L\left(\log ^{+} L\right)^{\alpha}\left(S^{m-1} \times S^{n-1}\right)$ (for $\left.\alpha>0\right)$ remains open. In 2006, Al-Qassem and Ali [13] gave the following result.

Theorem A ([13]) Let $\Gamma=\Phi=\left(\Phi_{1}, \Phi_{2}, \ldots, \Phi_{M}\right)$ and $\Upsilon=\Psi=\left(\Psi_{1}, \Psi_{2}, \ldots, \Psi_{N}\right)$ be two homogeneous mappings of degrees $d=\left(d_{1}, \ldots, d_{M}\right)$ and $v=\left(v_{1}, \ldots, v_{N}\right)$, respectively, with $d_{\iota}, v_{\kappa} \neq 0$ for $1 \leq \iota \leq M$ and $1 \leq \kappa \leq N$. Assume that $\left.\Gamma\right|_{S^{m-1}}$ and $\left.\Upsilon\right|_{S^{n-1}}$ are real-analytic. Suppose that $\Omega \in L\left(\log ^{+} L\right)^{2}\left(S^{m-1} \times S^{n-1}\right)$ satisfies (1.1). Then, for any $1<p<\infty$, there exists $C>0$ such that

$$
\left\|T_{\Omega, \Gamma, \Upsilon}(f)\right\|_{L^{p}\left(\mathbb{R}^{M} \times \mathbb{R}^{N}\right)} \leq C\|f\|_{L^{p}\left(\mathbb{R}^{M} \times \mathbb{R}^{N}\right)}
$$

for all $f \in L^{p}\left(\mathbb{R}^{M} \times \mathbb{R}^{N}\right)$.

A natural question, which arises from the above results, is the following:

Question 1.1 For $h \in \Delta_{\gamma}$ with $\gamma>1$, is $T_{h, \Omega, \Gamma, \Upsilon}$ bounded on $L^{p}\left(\mathbb{R}^{M} \times \mathbb{R}^{N}\right)$ under the same assumptions on $\Omega, \Gamma, \Upsilon$ as in Theorem A? 
Our investigation not only addresses this problem, but also deals with a more general class of operators. More specially, we shall establish the following:

Theorem 1.1 Let $\Gamma(y)=\Phi\left(\varphi(|y|) y^{\prime}\right)$ and $\Upsilon(y)=\Psi\left(\psi(|y|) y^{\prime}\right)$, where $\Phi=\left(\Phi_{1}, \Phi_{2}, \ldots, \Phi_{M}\right)$ and $\Psi=\left(\Psi_{1}, \Psi_{2}, \ldots, \Psi_{N}\right)$ are given as in Theorem $A$, and $\varphi, \psi \in \mathfrak{F}_{1}$ or $\mathfrak{F}_{2}$, where $\mathfrak{F}_{1}$ (resp., $\left.\mathfrak{F}_{2}\right)$ is the set of all functions $\phi$ satisfying the following condition (a) (resp., (b)):

(a) $\phi: \mathbb{R}_{+} \rightarrow \mathbb{R}_{+}$is an increasing $\mathcal{C}^{1}$ function such that $\phi^{\prime}$ is monotonous and $t \phi^{\prime}(t) \geq C_{\phi} \phi(t)$ and $\phi(2 t) \leq c_{\phi} \phi(t)$ for all $t>0$, where $C_{\phi}$ and $c_{\phi}$ are independent of $t$.

(b) $\phi: \mathbb{R}_{+} \rightarrow \mathbb{R}_{+}$is a decreasing $\mathcal{C}^{1}$ function such that $\phi^{\prime}$ is monotonous and $t \phi^{\prime}(t) \leq-C_{\phi} \phi(t)$ and $\phi(t) \leq c_{\phi} \phi(2 t)$ for all $t>0$, where $C_{\phi}$ and $c_{\phi}$ are independent of $t$.

Suppose that $h \in \Delta_{\gamma}$ for some $\gamma>1$ and $\Omega \in L\left(\log ^{+} L\right)^{2}\left(S^{m-1} \times S^{n-1}\right)$ satisfies (1.1). Then for $|1 / p-1 / 2|<\min \left\{1 / \gamma^{\prime}, 1 / 2\right\}$, there exists $C>0$ such that

$$
\left\|T_{h, \Omega, \Gamma, \Upsilon}(f)\right\|_{L^{p}\left(\mathbb{R}^{M} \times \mathbb{R}^{N}\right)} \leq C\|\Omega\|_{L\left(\log ^{+} L\right)^{2}\left(S^{m-1} \times S^{n-1}\right)}\|f\|_{L^{p}\left(\mathbb{R}^{M} \times \mathbb{R}^{N}\right)}
$$

for all $f \in L^{p}\left(\mathbb{R}^{M} \times \mathbb{R}^{N}\right)$.

Remark 1.1 Theorem 1.1 represents an improvement of the corresponding results in [1, 4 , 7]. Also, Theorem 1.1 implies Theorem A when $h \equiv 1$, even in the particular case $\varphi(t)=$ $\psi(t)=t$. There are some model examples for the class $\mathfrak{F}_{1}$, such as $t^{\alpha} \ln ^{\beta}(1+t)(\alpha>0, \beta \geq 0)$, $t \ln \ln (e+t)$, real-valued polynomials $P$ on $\mathbb{R}$ with positive coefficients and $P(0)=0$, and so on (see [14]). The model examples for a function $\varphi \in \mathfrak{F}_{2}$ are $t^{\delta}(\delta<0)$ and $t^{-1} \ln (1+1 / t)$. We point out that there exist a constant $B_{\varphi}>1$ such that $\varphi(2 t) \geq B_{\varphi} \varphi(t)\left(\right.$ or $\left.\varphi(t) \geq B_{\varphi} \varphi(2 t)\right)$ for $\varphi \in \mathfrak{F}_{1}$ (or $\mathfrak{F}_{2}$ ) (see [10]).

Remark 1.2 We remark that the one-parameter case of Theorem 1.1 was studied by many authors (see [15-18] for examples). It follows from Example 2.2 in [17] that Theorem 1.1 is not true if there exist $d_{i}=0$ or $v_{j}=0$ for some $i \in\{1,2, \ldots, M\}$ and $j \in\{1,2, \ldots, N\}$. It should be pointed out that the index 2 in $\Omega \in L\left(\log ^{+} L\right)^{2}\left(S^{m-1} \times S^{n-1}\right)$ is best possible since it has been shown in [3] that for any $\epsilon>0$, there is $\Omega \in L\left(\log ^{+} L\right)^{2-\epsilon}\left(S^{m-1} \times S^{n-1}\right)$ such that $T_{\Omega}$ is not bounded on $L^{p}\left(\mathbb{R}^{m} \times \mathbb{R}^{n}\right)$ for any $p \in(1, \infty)$.

When $\gamma \geq 2$, the range of $p$ in Theorem 1.1 is $(1, \infty)$, but for $1<\gamma<2$, the range of $p$ is shrunk to $\left(\frac{2 \gamma^{\prime}}{\gamma^{\prime}+2}, \frac{2 \gamma^{\prime}}{\gamma^{\prime}-2}\right)$. In light of the aforementioned facts, it is natural to ask the following question.

Question 1.2 Can the range of $p$ in Theorem 1.1 be enlarged for the case $1<\gamma<2$ ?

The next aim of this paper is to address this question by imposing some more restrictive conditions on $h$. Precisely, for $1 \leq \gamma \leq \infty$, let $U_{\gamma}\left(\mathbb{R}_{+} \times \mathbb{R}_{+}\right)$be the set of all measurable functions $h$ on $\mathbb{R}_{+} \times \mathbb{R}_{+}$satisfying

$$
\|h\|_{U_{\gamma}\left(\mathbb{R}_{+} \times \mathbb{R}_{+}\right)}:=\left(\int_{0}^{\infty} \int_{0}^{\infty}|h(r, s)|^{\gamma} \frac{d r d s}{r s}\right)^{1 / \gamma}<\infty
$$

For simplicity, we denote $U_{\gamma}\left(\mathbb{R}_{+} \times \mathbb{R}_{+}\right)$by $U_{\gamma}$ for $\gamma \geq 1$. Obviously, $U_{\gamma} \subsetneq \Delta_{\gamma}$ for $1<\gamma<\infty$ and $U_{\infty}=\Delta_{\infty}=L^{\infty}$. The second one of our main results can be formulated as follows. 
Theorem 1.2 Let $\Gamma, \Upsilon$ be as in Theorem 1.1. Suppose that $h \in U_{\gamma}$ for some $\gamma \geq 1$ and that $\Omega \in L\left(\log ^{+} L\right)^{2 / \gamma^{\prime}}\left(S^{m-1} \times S^{n-1}\right)$ satisfies (1.1). Then $T_{h, \Omega, \Gamma, \Upsilon}$ is bounded on $L^{p}\left(\mathbb{R}^{M} \times \mathbb{R}^{N}\right)$ if one of the following conditions holds:

(i) $\gamma=1, p=\infty$;

(ii) $\gamma>1,1<p<\infty$.

Remark 1.3 Obviously, when $\gamma=\infty$, Theorem 1.2 coincides with Theorem 1.1. When $1<\gamma<\infty$, the condition on $\Omega$ in Theorem 1.2 is strictly weaker than that in Theorem 1.1. Moreover, for $1<\gamma<2$, the range of $p$ in Theorem 1.2 is larger than that in Theorem 1.1. Meanwhile, we also obtain the result at the endpoint case $\gamma=1$. Therefore, it is worth to impose the above restriction on $h$. It is interesting, but not clear, whether the restriction on $h$ can be removed.

To prove Theorem 1.2, we need to consider the related maximal operators $M_{\Omega, \Gamma, \Upsilon}^{(\gamma)}$ defined by

$$
M_{\Omega, \Gamma, \Upsilon}^{(\gamma)} f(x, y)=\sup _{\|h\|_{U_{\gamma} \leq 1}}\left|T_{h, \Omega, \Gamma, \Upsilon} f(x, y)\right|
$$

which are interesting themselves. When $\Gamma(u)=u$ and $\Upsilon(v)=v$, we shall denote $M_{\Omega, \Gamma, \Upsilon}^{(\gamma)}$ by $M_{\Omega}^{(\gamma)}$. Historically, Ding [19] proved that the operator $M_{\Omega}^{(2)}$ is bounded on $L^{2}\left(\mathbb{R}^{m} \times \mathbb{R}^{n}\right)$, provided that $\Omega \in L\left(\log ^{+} L\right)^{2}\left(S^{m-1} \times S^{n-1}\right)$. This result was greatly improved by Al-Salman [20], who obtained the $L^{p}$ boundedness of $M_{\Omega}^{(2)}$ for $2 \leq p<\infty$ under the weaker condition that $\Omega \in L\left(\log ^{+} L\right)\left(S^{m-1} \times S^{n-1}\right)$. Moreover, Al-Salman showed that the condition $\Omega \in L\left(\log ^{+} L\right)\left(S^{m-1} \times S^{n-1}\right)$ cannot be replaced by any condition of the form $\Omega \in$ $L\left(\log ^{+} L\right)^{1-\epsilon}\left(S^{m-1} \times S^{n-1}\right), \epsilon>0$. Particularly, Al-Qassem and Pan [15] proved that the operator $M_{\Omega}^{(\gamma)}$ is bounded on $L^{p}\left(\mathbb{R}^{m} \times \mathbb{R}^{n}\right)$ for $\gamma^{\prime} \leq p<\infty$ (for $\gamma=1, p=\infty$ ) if $\Omega \in$ $L\left(\log ^{+} L\right)^{2 / \gamma^{\prime}}\left(S^{m-1} \times S^{n-1}\right)$ and $1 \leq \gamma \leq 2$ (also see [18] for the nonisotropic case).

The remaining main results can be formulated as follows.

Theorem 1.3 Let $\Gamma, \Upsilon$ be as in Theorem 1.1, and let $\Omega \in L\left(\log ^{+} L\right)^{2 / \gamma^{\prime}}\left(S^{m-1} \times S^{n-1}\right)$ for $1 \leq$ $\gamma \leq 2$ satisfy (1.1). Then $M_{\Omega, \Gamma, \Upsilon}^{(\gamma)}$ is bounded on $L^{p}\left(\mathbb{R}^{M} \times \mathbb{R}^{N}\right)$ for $1<\gamma \leq 2$ with $\gamma^{\prime} \leq p<\infty$, and it is bounded on $L^{\infty}\left(\mathbb{R}^{M} \times \mathbb{R}^{N}\right)$ for $\gamma=1$.

Remark 1.4 Theorem 1.3 improves and generalizes the results of $[19,20]$. Also, Theorem 1.3 generalizes the result of [15].

The rest of this paper is organized as follows. In Section 2, we shall recall some notation and establish some preliminary lemmas. The proofs of the main results will be given in Section 3. We remark that some ideas of our methods are taken from [4, 21], but our methods and technique are more delicate and complex than those used in $[4,15,21]$. Throughout this paper, let $p^{\prime}$ denote the conjugate index of $p$, that is, $1 / p+1 / p^{\prime}=1$. The letter $C$, sometimes with additional parameters, will stand for positive constants, not necessarily the same at each occurrence but independent of the essential variables. We also use the conventions $\sum_{j \in \emptyset} a_{j}=0$ and $\prod_{j \in \emptyset} a_{j}=1$. 


\section{Notation and auxiliary lemmas}

Following the notation in [13]. Let $\Omega \in L\left(\log ^{+} L\right)^{\alpha}\left(S^{m-1} \times S^{n-1}\right)$ satisfy (1.1) for some $\alpha>0$. For $\mu \in \mathbb{N}$, let $a_{\mu}=2^{\mu+1}, E_{\mu}=\left\{(x, y) \in S^{m-1} \times S^{n-1}: a_{\mu} / 2 \leq|\Omega(x, y)|<a_{\mu}\right\}, b_{\mu}=\Omega \chi_{E_{\mu}}$, and $\lambda_{\mu}=\left\|b_{\mu}\right\|_{1}$. Let $\mathcal{D}=\left\{\mu \in \mathbb{N}: \lambda_{\mu} \geq 2^{-3 \mu}\right\}$ and

$$
\begin{aligned}
\Omega_{\mu}(x, y)= & \lambda_{\mu}^{-1}\left(b_{\mu}(x, y)-\int_{S^{m-1}} b_{\mu}\left(u^{\prime}, y\right) d \sigma_{m}\left(u^{\prime}\right)-\int_{S^{n-1}} b_{\mu}\left(x, v^{\prime}\right) d \sigma_{n}\left(v^{\prime}\right)\right. \\
& \left.+\iint_{S^{m-1} \times S^{n-1}} b_{\mu}\left(u^{\prime}, v^{\prime}\right) d \sigma_{m}\left(u^{\prime}\right) d \sigma_{n}\left(v^{\prime}\right)\right)
\end{aligned}
$$

for $\mu \in \mathcal{D}$, and $\Omega_{0}=\Omega-\sum_{\mu \in \mathcal{D}} \lambda_{\mu} \Omega_{\mu}$. Let $\lambda_{0}=1$. Then

$$
\begin{aligned}
& \int_{S^{m-1}} \Omega_{\mu}\left(u^{\prime}, \cdot\right) d \sigma_{m}\left(u^{\prime}\right)=\int_{S^{n-1}} \Omega_{\mu}\left(\cdot, v^{\prime}\right) d \sigma_{n}\left(v^{\prime}\right)=0, \\
& \left\|\Omega_{\mu}\right\|_{L^{2}\left(S^{m-1} \times S^{n-1}\right)} \leq 4 a_{\mu}^{2} \quad \text { and } \quad\left\|\Omega_{\mu}\right\|_{L^{1}\left(S^{m-1} \times S^{n-1}\right)} \leq 4, \\
& \Omega(x, y)=\sum_{\mu \in \mathcal{D} \cup\{0\}} \lambda_{\mu} \Omega_{\mu}(x, y), \\
& \sum_{\mu \in \mathcal{D} \cup\{0\}} \lambda_{\mu}(\mu+1)^{\alpha} \leq C\|\Omega\|_{L\left(\log ^{+} L\right)^{\alpha}\left(S^{m-1} \times S^{n-1}\right)} \quad \text { for any } \alpha>0 .
\end{aligned}
$$

The following lemma of Van der Corput type was proved by Cheng [16].

Lemma 2.1 ([16]) Let $l \in \mathbb{N} \backslash\{0\}, \alpha_{1}, \ldots, \alpha_{l} \in \mathbb{R}$, and $v_{1}, \ldots, v_{l}$ be distinct nonzero real numbers. Let $\psi \in \mathcal{C}^{1}([0,1])$. Then there exists a positive constant $C$, independent of $\left\{\alpha_{j}\right\}_{j=1}^{l}$, such that

$$
\left|\int_{\delta}^{\tau} \exp \left(i\left(\alpha_{1} t^{\nu_{1}}+\cdots+\alpha_{l} t^{\nu_{l}}\right)\right) \psi(t) d t\right| \leq C\left|\alpha_{1}\right|^{-1 / l}\left(|\psi(\tau)|+\int_{\delta}^{\tau}\left|\psi^{\prime}(t)\right| d t\right)
$$

for $1 / 2 \leq \delta<\tau \leq 1$.

Applying Lemma 2.1, we have the following:

Lemma 2.2 Let $\Phi(t)=\mu_{1} t^{\alpha_{1}}+\mu_{2} t^{\alpha_{2}}+\cdots+\mu_{n} t^{\alpha_{n}}$, where $\mu_{1}, \mu_{2}, \ldots, \mu_{n} \in \mathbb{R}$, and $\alpha_{1}, \ldots, \alpha_{n}$ are distinct nonzero real numbers. Then for any $r>1$, there exists $C>0$ independent of $r$ such that

$$
\left|\int_{1}^{r} \exp (i(\Phi(t))) \frac{d t}{t}\right| \leq C\left(1+r^{-\alpha_{1} / n}\right)\left|\mu_{1}\right|^{-1 / n}
$$

Proof We can choose an integer $l \geq 0$ such that $2^{l} \leq r<2^{l+1}$. By the change of variables we have

$$
\begin{aligned}
\left|\int_{1}^{r} \exp (i(\Phi(t))) \frac{d t}{t}\right| & \leq \sum_{j=0}^{l-1}\left|\int_{2^{j}}^{2^{j+1}} \exp (i(\Phi(t))) \frac{d t}{t}\right|+\left|\int_{2^{l}}^{r} \exp (i(\Phi(t))) \frac{d t}{t}\right| \\
& \leq \sum_{j=0}^{l-1}\left|\int_{1 / 2}^{1} \exp \left(i\left(\Phi\left(2^{j+1} t\right)\right)\right) \frac{d t}{t}\right|+\left|\int_{\frac{2^{l}}{r}}^{1} \exp (i(\Phi(r t))) \frac{d t}{t}\right|
\end{aligned}
$$


Note that $1 / 2<2^{l} / r \leq 1$. Applying Lemma 2.1 and using (2.6), we obtain

$$
\begin{aligned}
\left|\int_{1}^{r} \exp (i(\Phi(t))) \frac{d t}{t}\right| & \leq C\left(\sum_{j=0}^{l-1}\left|\mu_{1}\right|^{-1 / n}\left(2^{j+1}\right)^{-\alpha_{1} / n}+\left|\mu_{1}\right|^{-1 / n} r^{-\alpha_{1} / n} \frac{r}{2^{l}}\right) \\
& \leq C\left(1+r^{-\alpha_{1} / n}\right)\left|\mu_{1}\right|^{-1 / n}
\end{aligned}
$$

where $C>0$ is independent of $r$. This proves Lemma 2.2.

Lemma 2.3 ([18]) Let $\Phi=\left(\Phi_{1}, \ldots, \Phi_{m}\right)$ be real analytic on $S^{n-1}$. Suppose that $\left\{\Phi_{1}, \ldots, \Phi_{m}\right\}$ is a linearly independent set. Then there exists a positive number $\delta=\delta(m, \Phi)$ such that

$$
\sup _{z \in S^{m-1}} \iint_{S^{n-1} \times S^{n-1}}|z \cdot(\Phi(x)-\Phi(y))|^{-\delta} d x d y<\infty .
$$

Let $\Gamma, \Upsilon, \Phi, \Psi, \varphi, \psi$ be as in Theorem 1.1. Let $\lambda_{1}$ be the number of distinct $d_{i}$, and $\lambda_{2}$ the number of distinct $v_{j}$. Without loss of generality, we may assume that

$$
\begin{aligned}
& \Phi=\left(\Phi^{1}, \Phi^{2}, \ldots, \Phi^{\lambda_{1}}\right), \\
& \Psi=\left(\Psi^{1}, \Psi^{2}, \ldots, \Psi^{\lambda_{2}}\right),
\end{aligned}
$$

where $\Phi^{\iota}=\left(\Phi_{\iota, 1}, \ldots, \Phi_{\iota, a_{\iota}}\right)$ with $\Phi_{\iota, i}(t y)=t^{d_{\alpha_{\iota}}} \Phi_{\iota, i}(y)$ for any $1 \leq \iota \leq \lambda_{1}$ and $1 \leq i \leq \iota$. and $\Psi^{\kappa}=\left(\Psi_{\kappa, 1}, \ldots, \Psi_{\kappa, b_{\kappa}}\right)$ with $\Psi_{\kappa, j}(t y)=t^{\nu_{\beta_{\kappa}}} \Psi_{\kappa, j}(y)$ for any $1 \leq \kappa \leq \lambda_{2}$ and $1 \leq j \leq \kappa$. Obviously, $\sum_{l=1}^{\lambda_{1}} a_{\iota}=M$ and $\left\{\alpha_{1}, \ldots, \alpha_{\lambda_{1}}\right\} \subset\{1, \ldots, M\}, \sum_{\kappa=1}^{\lambda_{2}} b_{\kappa}=M$ and $\left\{\beta_{1}, \ldots, \beta_{\lambda_{2}}\right\} \subset\{1, \ldots, N\}$. We also assume that $\left\{\Phi_{\iota, 1}, \ldots, \Phi_{\iota, o_{l}}\right\}$ forms a basis for $\operatorname{span}\left\{\Phi_{\iota, 1}, \ldots, \Phi_{\iota, a_{l}}\right\}$ for any $1 \leq \iota \leq \lambda_{1}$ and $\left\{\Psi_{\kappa, 1}, \ldots, \Psi_{\kappa, \varpi_{\kappa}}\right\}$ forms a basis for $\operatorname{span}\left\{\Psi_{\kappa, 1}, \ldots, \Psi_{\kappa, b_{\kappa}}\right\}$ for any $1 \leq \kappa \leq \lambda_{2}$. Thus, there exist two sequences of numbers $\left\{a_{l, i, l}\right\}$ and $\left\{b_{\kappa, j, k}\right\}$ such that

$$
\Phi_{l, i}(x)=a_{l, i, 1} \Phi_{l, 1}(x)+\cdots+a_{l, i, o_{l}} \Phi_{l, o_{l}}(x)
$$

for any $1 \leq \iota \leq \lambda_{1}, 1 \leq i \leq a_{\iota}$, and $x \in \mathbb{R}^{m}$ and

$$
\Psi_{\kappa, j}(y)=b_{\kappa, j, 1} \Psi_{\kappa, 1}(y)+\cdots+b_{\kappa, j, \varpi_{\kappa}} \Psi_{\kappa, \varpi_{\kappa}}(y)
$$

for any $1 \leq \kappa \leq \lambda_{2}, 1 \leq j \leq b_{\kappa}$, and $y \in \mathbb{R}^{n}$. Define two sequences of linear transformations $\left\{R_{l, i}\right\}_{i=1}^{o_{l}}: \mathbb{R}^{a_{l}} \rightarrow \mathbb{R}$ and $\left\{H_{\kappa, j}\right\}_{j=1}^{\omega_{\kappa}}: \mathbb{R}^{b_{\kappa}} \rightarrow \mathbb{R}$ as follows:

$$
\begin{aligned}
& R_{l, i}(y)=a_{\iota, 1, i} x_{1}+\cdots+a_{\iota, a_{l}, i} x_{a_{\iota}}, \quad 1 \leq i \leq o_{l} ; \\
& H_{\kappa, j}(y)=b_{\kappa, 1, j} y_{1}+\cdots+b_{\kappa, b_{\kappa}, j} y_{b_{\kappa}}, \quad 1 \leq j \leq \varpi_{\kappa} .
\end{aligned}
$$

Define two families of linear transformations $\left\{R_{l}\right\}_{l=1}^{\lambda_{1}}$ and $\left\{H_{\kappa}\right\}_{\kappa=1}^{\lambda_{2}}$ by

$$
\begin{aligned}
& R_{\iota}=\left(R_{\iota, 1}, \ldots, R_{\iota, o_{l}}\right), \\
& H_{\kappa}=\left(H_{\kappa, 1}, \ldots, H_{\kappa, \omega_{\kappa}}\right) .
\end{aligned}
$$


In what follows, let $\tilde{\Phi}^{\iota}=\left(\Phi_{\iota, 1}, \ldots, \Phi_{\iota, o_{l}}\right)$ and $\tilde{\Psi}^{\kappa}=\left(\Psi_{\kappa, 1}, \ldots, \Psi_{\kappa, \varpi_{\kappa}}\right)$. Let $\xi=\left(\xi_{1}, \ldots, \xi_{M}\right)=$ $\left(\xi^{1}, \ldots, \xi^{\lambda_{1}}\right)$ with $\xi^{\iota}=\left(\xi_{\iota, 1}, \xi_{\iota, 2}, \ldots, \xi_{l, a_{l}}\right)$ for any $1 \leq \iota \leq \lambda_{1}$. Let $\eta=\left(\eta_{1}, \ldots, \eta_{N}\right)=\left(\eta^{1}, \ldots, \eta^{\lambda_{2}}\right)$ with $\eta^{\kappa}=\left(\eta_{\kappa, 1}, \eta_{\kappa, 2}, \ldots, \eta_{\kappa, b_{\kappa}}\right)$ for any $1 \leq \kappa \leq \lambda_{2}$. Thus,

$$
\begin{aligned}
& \xi^{\iota} \cdot \Phi^{\iota}=R_{\iota}\left(\xi^{\iota}\right) \cdot \tilde{\Phi}^{\iota}, \quad 1 \leq \iota \leq \lambda_{1} \\
& \eta^{\kappa} \cdot \Psi^{\kappa}=H_{\kappa}\left(\eta^{\kappa}\right) \cdot \tilde{\Psi}^{\kappa}, \quad 1 \leq \kappa \leq \lambda_{2} .
\end{aligned}
$$

For $1 \leq \iota \leq \lambda_{1}$ and $1 \leq \kappa \leq \lambda_{2}$, we define two linear transformations $\mathcal{L}_{\iota}: \mathbb{R}^{M} \rightarrow \mathbb{R}^{o_{\iota}}$ and $\Theta_{\kappa}: \mathbb{R}^{N} \rightarrow \mathbb{R}^{\varpi_{\kappa}}$ by

$$
\mathcal{L}_{\iota}(\xi)=R_{\iota}\left(\xi^{\iota}\right), \quad \Theta_{\kappa}(\eta)=H_{\kappa}\left(\eta^{\kappa}\right)
$$

Define two families of mappings $\left\{\Gamma_{\iota}\right\}_{l=0}^{\lambda_{1}}$ and $\left\{\Upsilon_{\kappa}\right\}_{l=0}^{\lambda_{2}}$ by

$$
\begin{aligned}
& \Gamma_{0}=(0, \ldots, 0) ; \quad \Upsilon_{0}=(0, \ldots, 0) ; \\
& \Gamma_{\iota}=\left(\Phi^{1}, \ldots, \Phi^{\iota}, 0, \ldots, 0\right), \quad 1 \leq \iota<\lambda_{1} \quad \text { and } \quad \Gamma_{\lambda_{1}}=\left(\Phi^{1}, \ldots, \Phi^{\lambda_{1}}\right) \\
& \Upsilon_{\kappa}=\left(\Psi^{1}, \ldots, \Psi^{\kappa}, 0, \ldots, 0\right), \quad 1 \leq \kappa<\lambda_{2} \quad \text { and } \quad \Upsilon_{\lambda_{2}}=\left(\Psi^{1}, \ldots, \Psi^{\lambda_{2}}\right) .
\end{aligned}
$$

Let $\Omega_{\mu}$ be as in (2.1). For any $r, s>0$, define two families of measures $\left\{\sigma_{\mu ; r, s}^{\iota, \kappa}\right\}$ and $\left\{\left|\sigma_{\mu ; r, s}^{\iota, \kappa}\right|\right\}$ for $0 \leq \iota \leq \lambda_{1}$ and $0 \leq \kappa \leq \lambda_{2}$ as follows:

$$
\begin{aligned}
\widehat{\sigma_{\mu ;, r, s}^{\widehat{L, k}}}(\xi, \eta)= & \iint_{S^{m-1} \times S^{n-1}} \exp \left(-2 \pi i\left(\xi \cdot \Gamma_{\iota}\left(\varphi(r) u^{\prime}\right)\right.\right. \\
& \left.\left.+\eta \cdot \Upsilon_{\kappa}\left(\psi(s) v^{\prime}\right)\right)\right) \Omega_{\mu}\left(u^{\prime}, v^{\prime}\right) d \sigma_{m}\left(u^{\prime}\right) d \sigma_{n}\left(v^{\prime}\right),
\end{aligned}
$$

and $\left\{\left|\sigma_{\mu ; r, s}^{\ell, \kappa}\right|\right\}$ is defined in the same way as $\left\{\sigma_{\mu ; r, s}^{\iota, \kappa}\right\}$, but with $\Omega_{\mu}$ replaced by $\left|\Omega_{\mu}\right|$. Clearly,

$$
\sigma_{\mu ; r, s}^{0, \kappa}=\sigma_{\mu ; r, s}^{\iota, 0}=0 \text { for } 0 \leq \iota \leq \lambda_{1}, 0 \leq \kappa \leq \lambda_{2}
$$

Lemma 2.4 Let $\varphi, \psi \in \mathfrak{F}_{1}$ or $\mathfrak{F}_{2}$. For $\mu \in \mathcal{D} \cup\{0\}, k, j \in \mathbb{Z}, 1 \leq \iota \leq \lambda_{1}, 1 \leq \kappa \leq \lambda_{2}$, and $(\xi, \eta) \in \mathbb{R}^{M} \times \mathbb{R}^{N}$, there exist positive constants $\epsilon_{1}, \epsilon_{2}$, and $C$ such that

$$
\begin{aligned}
& \sup _{r, s>0}\left|\widehat{\sigma_{\mu, r, s}^{l, k}}(\xi, \eta)\right| \leq C \\
& \int_{a_{\mu}^{j}}^{d_{\mu}^{j+1}} \int_{a_{\mu}^{k}}^{a_{\mu}^{k+1}}\left|\widehat{\sigma_{\mu ;, r, s}^{l, k}}(\xi, \eta)-\widehat{\sigma_{\mu ; r, s}^{l-1, \kappa}}(\xi, \eta)\right|^{2} \frac{d r d s}{r s} \\
& \leq C(\mu+1)^{2}\left|\varphi\left(a_{\mu}^{k}\right)^{d \alpha_{l}} \mathcal{L}_{\iota}(\xi)\right|^{\frac{1}{\mu+1}} \min \left\{1,\left|\psi\left(a_{\mu}^{j}\right)^{v_{\beta_{\kappa}}} \Theta_{\kappa}(\eta)\right|^{-\frac{\epsilon_{2}}{\mu+1}}\right\} ; \\
& \int_{d_{\mu}^{j}}^{d_{\mu}^{j+1}} \int_{a_{\mu}^{k}}^{a_{\mu}^{k+1}}\left|\widehat{\sigma_{\mu ; r, s}, \widehat{l, k}}(\xi, \eta)-\widehat{\sigma_{\mu ; r, s}^{\widehat{l}, k-1}}(\xi, \eta)\right|^{2} \frac{d r d s}{r s} \\
& \leq C(\mu+1)^{2}\left|\psi\left(a_{\mu}^{j}\right)^{v_{\beta_{\kappa}}} \Theta_{\kappa}(\eta)\right|^{\frac{1}{\mu+1}} \min \left\{1,\left|\varphi\left(a_{\mu}^{k}\right)^{d \alpha_{l}} \mathcal{L}_{\iota}(\xi)\right|^{-\frac{\epsilon_{1}}{\mu+1}}\right\} ; \\
& \int_{d_{\mu}^{j}}^{d_{\mu}^{j+1}} \int_{a_{\mu}^{k}}^{a_{\mu}^{k+1}}\left|\widehat{\sigma_{\mu ;}, \widehat{k}, s}(\xi, \eta)\right|^{2} \frac{d r d s}{r s}
\end{aligned}
$$




$$
\begin{aligned}
& \leq C(\mu+1)^{2} \min \left\{1,\left|\varphi\left(a_{\mu}^{k}\right)^{d \alpha_{l}} \mathcal{L}_{l}(\xi)\right|^{-\frac{\epsilon_{1}}{\mu+1}},\right. \\
& \left.\left|\psi\left(a_{\mu}^{j}\right)^{v_{\beta_{\kappa}}} \Theta_{\kappa}(\eta)\right|^{-\frac{\epsilon_{2}}{\mu+1}},\left|\varphi\left(a_{\mu}^{k}\right)^{d \alpha_{\iota}} \mathcal{L}_{\iota}(\xi)\right|^{-\frac{\epsilon_{1}}{\mu+1}}\left|\psi\left(a_{\mu}^{j}\right)^{v_{\beta_{\kappa}}} \Theta_{\kappa}(\eta)\right|^{-\frac{\epsilon_{2}}{\mu+1}}\right\} ; \\
& \int_{d_{\mu}}^{d_{\mu}^{j+1}} \int_{a_{\mu}^{k}}^{a_{\mu}^{k+1}}\left|\widehat{\sigma_{\mu ; r, s}^{\widehat{L}, \kappa}}(\xi, \eta)-\widehat{\sigma_{\mu ; r, s}^{\widehat{l-1, \kappa}}}(\xi, \eta)-\widehat{\sigma_{\mu ; r, s}^{l, k-1}}(\xi, \eta)+\widehat{\sigma_{\mu ; r, s}^{l-1, \kappa-1}}(\xi, \eta)\right|^{2} \frac{d r d s}{r s} \\
& \leq C(\mu+1)^{2} \min \left\{1,\left|\varphi\left(a_{\mu}^{k}\right)^{d \alpha_{l}} \mathcal{L}_{l}(\xi)\right|^{\frac{1}{\mu+1}},\left|\psi\left(a_{\mu}^{j}\right)^{v_{\beta_{\kappa}}} \Theta_{\kappa}(\eta)\right|^{\frac{1}{\mu+1}},\right. \\
& \left.\left|\varphi\left(a_{\mu}^{k}\right)^{d \alpha_{\iota}} \mathcal{L}_{\iota}(\xi)\right|^{\frac{1}{\mu+1}}\left|\psi\left(a_{\mu}^{j}\right)^{v_{\beta_{\kappa}}} \Theta_{\kappa}(\eta)\right|^{\frac{1}{\mu+1}}\right\} .
\end{aligned}
$$

Proof Note that for any $\varphi \in \mathfrak{F}_{1}$ or $\mathfrak{F}_{2}$, there exist $C_{1}, C_{2}>0$ such that $C_{1}<\frac{\varphi(2 r)}{\varphi(r)}<C_{2}$. Thus, we only prove the case $\varphi, \psi \in \mathfrak{F}_{1}$, and other cases are analogous. Estimate (2.9) is obvious. By the change of variables,

$$
\begin{aligned}
& \left|\widehat{\sigma_{\mu ; r, s}^{\widehat{\tau}, k}}(\xi, \eta)\right|^{2} \\
& =\mid \iint_{S^{m-1} \times S^{n-1}} \exp \left(-2 \pi i\left(\xi \cdot \Gamma_{\iota}\left(\varphi(r) u^{\prime}\right)\right)\right. \\
& \left.+\eta \cdot \Upsilon_{\kappa}\left(\psi(s) v^{\prime}\right)\right)\left.\Omega_{\mu}\left(u^{\prime}, v^{\prime}\right) d \sigma_{m}\left(u^{\prime}\right) d \sigma_{n}\left(v^{\prime}\right)\right|^{2} \\
& =\iint_{\left(S^{m-1} \times S^{n-1}\right)^{2}} \exp \left(-2 \pi i\left(\xi \cdot\left(\Gamma_{\iota}\left(\varphi(r) u^{\prime}\right)-\Gamma_{\iota}(\varphi(r) \theta)\right)\right)\right) \\
& \times \exp \left(-2 \pi i \eta \cdot\left(\Upsilon_{\kappa}\left(\psi(s) v^{\prime}\right)-\Upsilon_{\kappa}(\psi(s) \omega)\right)\right) \\
& \times \Omega_{\mu}\left(u^{\prime}, v^{\prime}\right) \overline{\Omega_{\mu}(\theta, \omega)} d \sigma_{m}\left(u^{\prime}\right) d \sigma_{n}\left(v^{\prime}\right) d \sigma_{m}(\theta) d \sigma_{n}(\omega) .
\end{aligned}
$$

It follows that

$$
\begin{aligned}
& \int_{d_{\mu}^{j}}^{d_{\mu}^{j+1}} \int_{a_{\mu}^{k}}^{a_{\mu}^{k+1}} \mid \widehat{\left.\sigma_{\mu ; r, s}^{\widehat{l, k}}(\xi, \eta)\right|^{2}} \frac{d r d s}{r s} \\
& \leq \iint_{\left(S^{m-1} \times S^{n-1}\right)^{2}}\left|H_{k, \mu}\left(u^{\prime}, \theta, \xi\right)\right|\left|J_{j, \mu}\left(v^{\prime}, \omega, \eta\right)\right| \\
& \quad \times\left|\Omega_{\mu}\left(u^{\prime}, v^{\prime}\right) \overline{\Omega_{\mu}(\theta, \omega)}\right| d \sigma_{m}\left(u^{\prime}\right) d \sigma_{n}\left(v^{\prime}\right) d \sigma_{m}(\theta) d \sigma_{n}(\omega)
\end{aligned}
$$

where

$$
\begin{aligned}
& H_{k, \mu}\left(u^{\prime}, \theta, \xi\right):=\int_{a_{\mu}^{k}}^{a_{\mu}^{k+1}} \exp \left(-2 \pi i \xi \cdot\left(\Gamma_{\iota}\left(\varphi(r) u^{\prime}\right)-\Gamma_{\iota}(\varphi(r) \theta)\right)\right) \frac{d r}{r} \\
& J_{j, \mu}\left(v^{\prime}, \omega, \eta\right):=\int_{a_{\mu}^{j}}^{d_{\mu}^{j+1}} \exp \left(-2 \pi i \xi \cdot\left(\Upsilon_{\kappa}\left(\psi(s) v^{\prime}\right)-\Upsilon_{\kappa}(\psi(s) \omega)\right)\right) \frac{d s}{s} .
\end{aligned}
$$

By the change of variables and (2.7) we have

$$
\begin{aligned}
& \left|H_{k, \mu}\left(u^{\prime}, \theta, \xi\right)\right| \\
& \quad=\left|\sum_{l=0}^{\mu} \int_{2^{l} a_{\mu}^{k}}^{2^{l+1} a_{\mu}^{k}} \exp \left(-2 \pi i \xi \cdot\left(\Gamma_{\iota}\left(\varphi(r) u^{\prime}\right)-\Gamma_{\iota}(\varphi(r) \theta)\right)\right) \frac{d r}{r}\right|
\end{aligned}
$$




$$
\begin{aligned}
& =\sum_{l=0}^{\mu} \mid \int_{\varphi\left(2^{l} a_{\mu}^{k}\right)}^{\varphi\left(2^{l+1} a_{\mu}^{k}\right)} \exp \left(-2 \pi i \xi \cdot\left(\Gamma_{\iota}\left(r u^{\prime}\right)-\Gamma_{\iota}(r \theta)\right) \frac{d r}{r} \mid\right. \\
& =\sum_{l=0}^{\mu}\left|\int_{1}^{\frac{\left.\varphi 2^{l+1} a_{\mu}^{k}\right)}{\varphi\left(2^{l} a_{\mu}^{k}\right)}} \exp \left(-2 \pi i \xi \cdot\left(\Gamma_{\iota}\left(\varphi\left(2^{l} a_{\mu}^{k}\right) r u^{\prime}\right)-\Gamma_{\iota}\left(\varphi\left(2^{l} a_{\mu}^{k}\right) r \theta\right)\right)\right) \frac{d r}{r}\right| \\
& =\sum_{l=0}^{\mu}\left|\int_{1}^{\frac{\varphi\left(2^{l+1} a_{\mu}^{k}\right)}{\varphi\left(2^{l} a_{\mu}^{k}\right)}} \exp \left(-2 \pi i \sum_{j=1}^{l} \varphi\left(2^{l} a_{\mu}^{k}\right)^{d \alpha_{j}} r^{d \alpha_{j}} R_{j}\left(\xi^{j}\right) \cdot\left(\tilde{\Phi}^{j}\left(u^{\prime}\right)-\tilde{\Phi}^{j}(\theta)\right)\right) \frac{d r}{r}\right| .
\end{aligned}
$$

Note that $\frac{\varphi\left(2^{l+1} a_{\mu}^{k}\right)}{\varphi\left(2^{l} a_{\mu}^{k}\right)} \leq c_{\varphi}$. Combining (2.15) with Lemma 2.2 yields that

$$
\begin{aligned}
\left|H_{k, \mu}\left(u^{\prime}, \theta, \xi\right)\right| \leq & \sum_{l=0}^{\mu} \min \left\{1,\left|\varphi\left(2^{l} a_{\mu}^{k}\right)^{d \alpha_{j}} R_{\iota}\left(\xi^{\iota}\right) \cdot\left(\tilde{\Phi}^{\iota}\left(u^{\prime}\right)-\tilde{\Phi}^{\iota}(\theta)\right)\right|^{-1 / \iota}\right\} \\
\leq & C(\mu+1) \min \left\{1, \max \left\{\varphi\left(a_{\mu}^{k}\right)^{d \alpha_{j}}, \varphi\left(2^{\mu} a_{\mu}^{k}\right)^{d \alpha_{j}}\right\}^{-\epsilon_{1}}\right. \\
& \left.\times\left|R_{\iota}\left(\xi^{\iota}\right) \cdot\left(\tilde{\Phi}^{\iota}\left(u^{\prime}\right)-\tilde{\Phi}^{\iota}(\theta)\right)\right|^{-\epsilon_{1}}\right\}
\end{aligned}
$$

for any $0<\epsilon_{1} \leq 1 / \lambda_{1}$. Similarly, we have

$$
\begin{aligned}
\left|J_{j, \mu}\left(v^{\prime}, \omega, \eta\right)\right| \leq & C(\mu+1) \min \left\{1, \max \left\{\psi\left(a_{\mu}^{j}\right)^{v_{\beta_{\kappa}}}, \psi\left(2^{\mu} a_{\mu}^{j}\right)^{v_{\beta_{\kappa}}}\right\}^{-\epsilon_{2}}\right. \\
& \left.\times\left|H_{\kappa}\left(\eta^{\kappa}\right) \cdot\left(\tilde{\Psi}^{\kappa}\left(v^{\prime}\right)-\tilde{\Psi}^{\kappa}(\omega)\right)\right|^{-\epsilon_{2}}\right\}
\end{aligned}
$$

for any $0<\epsilon_{2} \leq 1 / \lambda_{2}$. For any $1 \leq \iota \leq \lambda_{1}$ and $x \in S^{o_{t}-1}$, since $\left\{\Phi_{\iota, 1}, \ldots, \Phi_{\iota, o_{l}}\right\}$ is linear independent, $x \cdot \tilde{\Phi}^{l}(\cdot)$ is a nonzero real-analytic function. Invoking Lemma 2.3 , there exists $\delta_{1}>0$ such that

$$
\sup _{x \in S^{o^{\prime}-1}} \iint_{\left(S^{m-1}\right)^{2}}\left|x \cdot\left(\tilde{\Phi}^{\iota}\left(u^{\prime}\right)-\tilde{\Phi}^{\iota}(\theta)\right)\right|^{-\delta_{1}} d \sigma_{m}\left(u^{\prime}\right) d \sigma_{m}(\theta)<\infty
$$

Similarly, for any $1 \leq \kappa \leq \lambda_{2}$, there exists $\delta_{2}>0$ such that

$$
\sup _{y \in S^{\sigma_{\kappa}-1}} \iint_{\left(S^{n-1}\right)^{2}}\left|y \cdot\left(\tilde{\Psi}^{\kappa}\left(u^{\prime}\right)-\tilde{\Psi}^{\kappa}(\theta)\right)\right|^{-\delta_{2}} d \sigma_{n}\left(u^{\prime}\right) d \sigma_{n}(\theta)<\infty .
$$

From (2.14) and (2.16)-(2.17) we have

$$
\begin{aligned}
& \int_{a_{\mu}^{j}}^{a_{\mu}^{j+1}} \int_{a_{\mu}^{k}}^{a_{\mu}^{k+1}}\left|\widehat{\sigma_{\mu ; r, s}^{l, k}}(\xi, \eta)\right|^{2} \frac{d r d s}{r s} \\
& \leq C(\mu+1)^{2} \min \left\{1, \max \left\{\varphi\left(a_{\mu}^{k}\right)^{d \alpha_{j}}, \varphi\left(2^{\mu} a_{\mu}^{k}\right)^{d \alpha_{j}}\right\}^{-\epsilon_{1}}\left|R_{\iota}\left(\xi^{\iota}\right)\right|^{-\epsilon_{1}}\right\} \\
& \times \min \left\{1, \max \left\{\psi\left(a_{\mu}^{j}\right)^{v_{\beta_{\kappa}}}, \psi\left(2^{\mu} a_{\mu}^{j}\right)^{v_{\beta_{\kappa}}}\right\}^{-\epsilon_{2}}\left|H_{\kappa}\left(\eta^{\kappa}\right)\right|^{-\epsilon_{2}}\right\} \\
& \times \iint_{\left(S^{m-1} \times S^{n-1}\right)^{2}}\left|\frac{R_{\iota}\left(\xi^{\iota}\right)}{\left|R_{\iota}\left(\xi^{\iota}\right)\right|} \cdot\left(\tilde{\Phi}^{\iota}\left(u^{\prime}\right)-\tilde{\Phi}^{\iota}(\theta)\right)\right|^{-\epsilon_{1}}\left|\frac{H_{\kappa}\left(\eta^{\kappa}\right)}{\left|H_{\kappa}\left(\eta^{\kappa}\right)\right|} \cdot\left(\tilde{\Psi}^{\kappa}\left(v^{\prime}\right)-\tilde{\Psi}^{\kappa}(\omega)\right)\right|^{-\epsilon_{2}} \\
& \times\left|\Omega_{\mu}\left(u^{\prime}, v^{\prime}\right) \overline{\Omega_{\mu}(\theta, \omega)}\right| d \sigma_{m}\left(u^{\prime}\right) d \sigma_{n}\left(v^{\prime}\right) d \sigma_{m}(\theta) d \sigma_{n}(\omega)
\end{aligned}
$$


for any $0<\epsilon_{1} \leq 1 / \lambda_{1}$ and $0<\epsilon_{2} \leq 1 / \lambda_{2}$. Take $\epsilon_{1}=\min \left\{1 / \lambda_{1}, \delta_{1} / 2\right\}$ and $\epsilon_{2}=\min \left\{1 / \lambda_{2}, \delta_{2} / 2\right\}$. Using (2.3), (2.18)-(2.19) and Hölder's inequality along with (2.20), we obtain

$$
\begin{aligned}
\int_{d_{\mu}^{j}}^{d_{\mu}^{j+1}} \int_{a_{\mu}^{k}}^{a_{\mu}^{k+1}}\left|\widehat{\sigma_{\mu ; r, s}^{\iota,}}(\xi, \eta)\right|^{2} \frac{d r d s}{r s} \\
\leq C a_{\mu}^{4}(\mu+1)^{2} \min \left\{1, \max \left\{\varphi\left(a_{\mu}^{k}\right)^{d \alpha_{j}}, \varphi\left(2^{\mu} a_{\mu}^{k}\right)^{d \alpha_{j}}\right\}^{-\epsilon_{1}}\left|R_{\iota}\left(\xi^{\iota}\right)\right|^{-\epsilon_{1}}\right\} \\
\quad \times \min \left\{1, \max \left\{\psi\left(a_{\mu}^{j}\right)^{v_{\beta_{\kappa}}}, \psi\left(2^{\mu} a_{\mu}^{j}\right)^{v_{\beta_{\kappa}}}\right\}^{-\epsilon_{2}}\left|H_{\kappa}\left(\eta^{\kappa}\right)\right|^{-\epsilon_{2}}\right\} .
\end{aligned}
$$

We can easily check that

$$
\begin{aligned}
& \int_{a_{\mu}^{j}}^{d_{\mu}^{j+1}} \int_{a_{\mu}^{k}}^{a_{\mu}^{k+1}}\left|\widehat{\sigma_{\mu ; r, r}, k}(\xi, \eta)\right|^{2} \frac{d r d s}{r s} \leq C(\mu+1)^{2} \\
& \int_{a_{\mu}^{j}}^{d_{\mu}^{j+1}} \int_{a_{\mu}^{k}}^{a_{\mu}^{k+1}}\left|\widehat{\sigma_{\mu ; r, s}(\xi,}(\xi, \eta)-\widehat{\sigma_{\mu ; r, s}^{l-1, \kappa}}(\xi, \eta)\right|^{2} \frac{d r d s}{r s} \leq C(\mu+1)^{2} .
\end{aligned}
$$

Interpolating between (2.21) and (2.22) yields (2.12). We now prove (2.10) and (2.11). By the change of variables,

$$
\begin{aligned}
& \left|\widehat{\sigma_{\mu ; r, s}^{l, k}}(\xi, \eta)-\widehat{\sigma_{\mu ; r, s}^{l-1, \kappa}}(\xi, \eta)\right|^{2} \\
& =\mid \iint_{S^{m-1} \times S^{n-1}}\left(\exp \left(-2 \pi i \xi \cdot \Gamma_{\iota}\left(\varphi(r) u^{\prime}\right)\right)-\exp \left(-2 \pi i \xi \cdot \Gamma_{\iota-1}\left(\varphi(r) u^{\prime}\right)\right)\right) \\
& \times\left.\exp \left(-2 \pi i \eta \cdot \Upsilon_{\kappa}\left(\psi(s) v^{\prime}\right)\right) \Omega_{\mu}\left(u^{\prime}, v^{\prime}\right) d \sigma_{m}\left(u^{\prime}\right) d \sigma_{n}\left(v^{\prime}\right)\right|^{2} \\
& =\iint_{\left(S^{m-1} \times S^{n-1}\right)^{2}}\left(\exp \left(-2 \pi i \xi \cdot \Gamma_{\iota}\left(\varphi(r) u^{\prime}\right)\right)-\exp \left(-2 \pi i \xi \cdot \Gamma_{\iota-1}\left(\varphi(r) u^{\prime}\right)\right)\right) \\
& \times\left(\exp \left(2 \pi i \xi \cdot \Gamma_{\iota}(\varphi(r) \theta)\right)-\exp \left(-2 \pi i \xi \cdot \Gamma_{\iota-1}(\varphi(r) \theta)\right)\right) \\
& \times \exp \left(-2 \pi i \eta \cdot\left(\Upsilon_{\kappa}\left(\psi(s) v^{\prime}\right)-\Upsilon_{\kappa}(\psi(s) \omega)\right)\right) \\
& \times \Omega_{\mu}\left(u^{\prime}, v^{\prime}\right) \overline{\Omega_{\mu}(\theta, \omega)} d \sigma_{m}\left(u^{\prime}\right) d \sigma_{n}\left(v^{\prime}\right) d \sigma_{m}(\theta) d \sigma_{n}(\omega),
\end{aligned}
$$

which, together with (2.7), implies that

$$
\begin{aligned}
& \int_{a_{\mu}^{j}}^{a_{\mu}^{j+1}} \int_{a_{\mu}^{k}}^{a_{\mu}^{k+1}}\left|\widehat{\sigma_{\mu ; r, s}^{\widehat{l, k}}}(\xi, \eta)-\widehat{\sigma_{\mu ; r, s}^{\widehat{l-1, \kappa}}}(\xi, \eta)\right|^{2} \frac{d r d s}{r s} \\
& \leq C(\mu+1) \min \left\{1,\left|\varphi\left(a_{\mu}^{k}\right)^{d_{\alpha_{l}}} R_{\iota}\left(\xi^{\iota}\right)\right|\right\} \iint_{\left(S^{m-1} \times S^{n-1}\right)^{2}}\left|J_{j, \mu}\left(v^{\prime}, \omega, \eta\right)\right| \\
& \quad \times\left|\Omega_{\mu}\left(u^{\prime}, v^{\prime}\right) \overline{\Omega_{\mu}(\theta, \omega)}\right| d \sigma_{m}\left(u^{\prime}\right) d \sigma_{n}\left(v^{\prime}\right) d \sigma_{m}(\theta) d \sigma_{n}(\omega) .
\end{aligned}
$$


Using (2.3), (2.17), (2.19), (2.24), and Hölder's inequality, we have

$$
\begin{aligned}
& \int_{a_{\mu}^{j}}^{d_{\mu}^{j+1}} \int_{a_{\mu}^{k}}^{a_{\mu}^{k+1}}\left|\widehat{\sigma_{\mu ; r, s}^{\widehat{l, k}}}(\xi, \eta)-\widehat{\sigma_{\mu ; r, s}^{l-1, \kappa}}(\xi, \eta)\right|^{2} \frac{d r d s}{r s} \\
& \leq C a_{\mu}^{4}(\mu+1)^{2} \min \left\{1,\left|\varphi\left(a_{\mu}^{k}\right)^{d_{\alpha_{l}}} R_{\iota}\left(\xi^{\iota}\right)\right|\right\} \\
& \quad \times \min \left\{1, \max \left\{\psi\left(a_{\mu}^{j}\right)^{v_{\beta_{\kappa}}}, \psi\left(2^{\mu} a_{\mu}^{j}\right)^{v_{\beta_{\kappa}}}\right\}^{-\epsilon_{2}}\left|H_{\kappa}\left(\eta^{\kappa}\right)\right|^{-\epsilon_{2}}\right\} .
\end{aligned}
$$

Estimate (2.10) follows form (2.23) and (2.25). Similarly, we can get (2.11). Estimate (2.13) follows from the inequality

$$
\begin{aligned}
& \left|\widehat{\sigma_{\mu ; r, s}^{\widehat{l, \kappa}}}(\xi, \eta)-\widehat{\sigma_{\mu ; r, s}^{\iota-1, \kappa}}(\xi, \eta)-\widehat{\sigma_{\mu ; r, s}^{\iota, \kappa-1}}(\xi, \eta)+\widehat{\sigma_{\mu ; r, s}^{\iota-1, \kappa-1}}(\xi, \eta)\right| \\
& =\mid \iint_{S^{m-1} \times S^{n-1}} \exp \left(-2 \pi i\left(\xi \cdot \Gamma_{\iota-1}\left(\varphi(r) u^{\prime}\right)+\eta \cdot \Upsilon_{\kappa-1}\left(\psi(s) v^{\prime}\right)\right)\right) \\
& \quad \times\left(\exp \left(-2 \pi i\left(\xi \cdot\left(\Gamma_{\iota}\left(\varphi(r) u^{\prime}\right)-\Gamma_{\iota-1}\left(\varphi(r) u^{\prime}\right)\right)\right)\right)-1\right) \\
& \quad \times\left(\exp \left(-2 \pi i\left(\eta \cdot\left(\Upsilon_{\kappa}\left(\psi(s) v^{\prime}\right)-\Upsilon_{\kappa-1}\left(\psi(s) v^{\prime}\right)\right)\right)\right)-1\right) \\
& \quad \times \Omega\left(u^{\prime}, v^{\prime}\right) J_{m}\left(u^{\prime}\right) J_{n}\left(v^{\prime}\right) d \sigma_{m}\left(u^{\prime}\right) d \sigma_{n}\left(v^{\prime}\right) \mid \\
& \leq C \min \left\{1,\left|\varphi(r)^{d_{\alpha_{\iota}}} R_{\iota}\left(\xi^{\iota}\right)\right|\right\} \min \left\{1,\left|\psi(s)^{v_{\beta_{\kappa}}} H_{\kappa}\left(\eta^{\kappa}\right)\right|\right\} .
\end{aligned}
$$

This proves Lemma 2.4 .

For any $\mu \in \mathcal{D} \cup\{0\}$ and $k, j \in \mathbb{Z}$, we define two families of measures $\left\{\tau_{\mu ; k, j}^{\iota, \kappa}\right\}$ and $\left\{\left|\tau_{\mu ; k, j}^{\iota, \kappa}\right|\right\}$ for $1 \leq \iota \leq \lambda_{1}$ and $1 \leq \kappa \leq \lambda_{2}$ as follows:

$$
\begin{aligned}
\widehat{\tau_{\mu ; k, j}^{l, \kappa}}(\xi, \eta)= & \iint_{\triangle_{\mu ; k, j}} \frac{\Omega_{\mu}\left(u^{\prime}, v^{\prime}\right) h(|u|,|v|)}{|u|^{m}|v|^{n}} \exp \left(-2 \pi i\left(\xi \cdot \Gamma_{\iota}\left(\varphi(|u|) u^{\prime}\right)\right.\right. \\
& \left.\left.+\eta \cdot \Upsilon_{\kappa}\left(\psi(|v|) v^{\prime}\right)\right)\right) d u d v, \\
\widehat{\left|\tau_{\mu ; k, j}^{l, k}\right|}(\xi, \eta)= & \iint_{\triangle_{\mu ; k, j}} \frac{\left|\Omega_{\mu}\left(u^{\prime}, v^{\prime}\right) h(|u|,|v|)\right|}{|u|^{m}|v|^{n}} \exp \left(-2 \pi i\left(\xi \cdot \Gamma_{\iota}\left(\varphi(|u|) u^{\prime}\right)\right.\right. \\
& \left.\left.+\eta \cdot \Upsilon_{\kappa}\left(\psi(|v|) v^{\prime}\right)\right)\right) d u d v,
\end{aligned}
$$

where $(\xi, \eta) \in \mathbb{R}^{M} \times \mathbb{R}^{N}$ and

$$
\triangle_{\mu ; k, j}=\left\{(u, v) \in \mathbb{R}^{m} \times \mathbb{R}^{n}:(|u|,|v|)\left[a_{\mu}^{k}, a_{\mu}^{k+1}\right) \times\left[a_{\mu}^{j}, a_{\mu}^{j+1}\right)\right\}
$$

Observe that

$$
\tau_{\mu ; k, j}^{0, \kappa}=\tau_{\mu ; k, j}^{\iota, 0}=0 \quad \text { for } 0 \leq \iota \leq \lambda_{1}, 0 \leq \kappa \leq \lambda_{2}
$$

For convenience, for $\mu \in \mathcal{D} \cup\{0\}$ and $\lambda>1$, we set

$$
\|h\|_{\mu, \gamma}:=\sup _{j, k \in \mathbb{Z}}\left(\int_{a_{\mu}^{j}}^{a_{\mu}^{j+1}} \int_{a_{\mu}^{k}}^{a_{\mu}^{k+1}}|h(r, s)|^{\gamma} \frac{d r d s}{r s}\right)^{1 / \gamma}
$$


Lemma 2.5 Let $\mu \in \mathcal{D} \cup\{0\},\|h\|_{\mu, \gamma}<\infty$ for some $\gamma>1$ and $\tilde{\gamma}=\max \left\{2, \gamma^{\prime}\right\}$. Suppose $\varphi, \psi \in$ $\mathfrak{F}_{1}$ or $\mathfrak{F}_{2}$. Then for $k, j \in \mathbb{Z}$, there exist positive constants $\epsilon_{1}, \epsilon_{2}, C$ such that, for any $1 \leq \iota \leq \lambda_{1}$ and $1 \leq \kappa \leq \lambda_{2}$,

$$
\begin{aligned}
& \left|\widehat{\tau_{\mu ; k, j}^{\widehat{l, k}}}(\xi, \eta)\right| \\
& \leq C\|h\|_{\mu, \gamma}(\mu+1)^{2 / \gamma^{\prime}} \min \left\{1,\left|\varphi\left(a_{\mu}^{k}\right)^{d_{\alpha_{l}}} \mathcal{L}_{l}(\xi)\right|^{-\frac{\epsilon_{1}}{\gamma}(\mu+1)},\left|\psi\left(a_{\mu}^{j}\right)^{v_{\beta_{K}}} \Theta_{\kappa}(\eta)\right|^{-\frac{\epsilon_{2}}{\gamma(\mu+1)}},\right. \\
& \left.\left|\varphi\left(a_{\mu}^{k}\right)^{d_{\alpha_{l}}} \mathcal{L}_{l}(\xi)\right|^{-\frac{\epsilon_{1}}{\gamma(\mu+1)}}\left|\psi\left(a_{\mu}^{j}\right)^{v_{\beta_{\kappa}}} \Theta_{\kappa}(\eta)\right|^{-\frac{\epsilon_{2}}{\gamma(\mu+1)}}\right\} ; \\
& \left|\widehat{\tau_{\mu ; k, j}}(\xi, \eta)-\widehat{\tau_{\mu ; k, j}^{\imath, k-1}}(\xi, \eta)\right| \\
& \leq C\|h\|_{\mu, \gamma}(\mu+1)^{2 / \gamma^{\prime}}\left|\psi\left(a_{\mu}^{j}\right)^{v_{\beta_{\kappa}}} \Theta_{\kappa}(\eta)\right|^{\frac{1}{\bar{\gamma}(\mu+1)}} \min \left\{1,\left|\varphi\left(a_{\mu}^{k}\right)^{d_{\alpha_{l}}} \mathcal{L}_{l}(\xi)\right|^{-\frac{\epsilon_{1}}{\bar{\gamma}(\mu+1)}}\right\} ; \\
& \left|\widehat{\tau_{\mu ; k, j}^{l, k}}(\xi, \eta)-\widehat{\tau_{\mu ; k, j}^{l-1, \kappa}}(\xi, \eta)\right| \\
& \leq C\|h\|_{\mu, \gamma}(\mu+1)^{2 / \gamma^{\prime}}\left|\varphi\left(a_{\mu}^{k}\right)^{d_{\alpha_{l}}} \mathcal{L}_{l}(\xi)\right|^{\frac{1}{\bar{\gamma}(\mu+1)}} \min \left\{1,\left|\psi\left(a_{\mu}^{j}\right)^{v_{\beta_{k}}} \Theta_{\kappa}(\eta)\right|^{-\frac{\epsilon_{2}}{\bar{\gamma}(\mu+1)}}\right\} ; \\
& \left|\widehat{\tau_{\mu ; k, j}^{\imath, \kappa}}(\xi, \eta)-\widehat{\tau_{\mu ; k, j}^{\imath-1, k}}(\xi, \eta)-\widehat{\tau_{\mu ; k, j}^{l, k-1}}(\xi, \eta)+\widehat{\tau_{\mu ; k, j}^{l-1, k-1}}(\xi, \eta)\right| \\
& \leq C\|h\|_{\mu, \gamma}(\mu+1)^{2 / \gamma^{\prime}} \min \left\{1,\left|\varphi\left(a_{\mu}^{k}\right)^{d_{\alpha_{l}}} \mathcal{L}_{l}(\xi)\right|^{\frac{1}{\tilde{\gamma}(\mu+1)}},\right. \\
& \left.\left|\psi\left(a_{\mu}^{j}\right)^{v_{\beta_{\kappa}}} \Theta_{\kappa}(\eta)\right|^{\frac{1}{\hat{\gamma}(\mu+1)}},\left|\varphi\left(a_{\mu}^{k}\right)^{d_{\alpha_{l}}} \mathcal{L}_{l}(\xi)\right|^{\frac{1}{\tilde{\gamma}(\mu+1)}}\left|\psi\left(a_{\mu}^{j}\right)^{v_{\beta_{\kappa}}} \Theta_{\kappa}(\eta)\right|^{\frac{1}{\bar{\gamma}(\mu+1)}}\right\} .
\end{aligned}
$$

Proof By a change of variables, (2.9), and Hölder's inequality we have

$$
\begin{aligned}
& \left|\widehat{\tau_{\mu, k, j}^{\iota, \kappa}}(\xi, \eta)\right|=\mid \int_{d_{\mu}^{j}}^{d_{\mu}^{j+1}} \int_{a_{\mu}^{k}}^{a_{\mu}^{k+1}} \iint_{S^{m-1} \times S^{n-1}} \exp \left(-2 \pi i\left(\xi \cdot \Gamma_{\iota}\left(\varphi(r) u^{\prime}\right)+\eta \cdot \Upsilon_{\kappa}\left(\psi(s) v^{\prime}\right)\right)\right) \\
& \times \Omega_{\mu}\left(u^{\prime}, v^{\prime}\right) d \sigma_{m}\left(u^{\prime}\right) d \sigma_{n}\left(v^{\prime}\right) h(r, s) \frac{d r d s}{r s} \mid \\
& \leq C\|h\|_{\mu, \gamma}\left(\int_{d_{\mu}^{j}}^{d_{\mu}^{j+1}} \int_{a_{\mu}^{k}}^{a_{\mu}^{k+1}}\left|\widehat{\sigma_{\mu ; r, s}} \widehat{\phi, k}(\xi, \eta)\right|^{\gamma^{\prime}} \frac{d r d s}{r s}\right)^{1 / \gamma^{\prime}} \\
& \leq C\|h\|_{\mu, \gamma}(\mu+1)^{2 \max \left\{1 / \gamma^{\prime}-1 / 2,0\right\}}\left(\int_{a_{\mu}^{j}}^{a_{\mu}^{j+1}} \int_{a_{\mu}^{k}}^{a_{\mu}^{k+1}}\left|\widehat{\sigma_{\mu ;, r, s}}(\xi, \eta)\right|^{2} \frac{d r d s}{r s}\right)^{1 / \tilde{\gamma}}
\end{aligned}
$$

This inequality, together with (2.12), yields (2.27). By a change of variables again, (2.9) and Hölder's inequality we obtain

$$
\begin{aligned}
& \left|\widehat{\tau_{\mu, k, j}^{\imath, k}}(\xi, \eta)-\widehat{\tau_{\mu, k, j}^{l, k-1}}(\xi, \eta)\right| \\
& =\mid \int_{d_{\mu}^{j}}^{a_{\mu}^{j+1}} \int_{a_{\mu}^{k}}^{a_{\mu}^{k+1}} \iint_{S^{m-1} \times S^{n-1}} \exp \left(-2 \pi i \xi \cdot \Gamma_{\iota}\left(\varphi(r) u^{\prime}\right)\right) \\
& \quad \times\left(\exp \left(-2 \pi i \eta \cdot \Upsilon_{\kappa}\left(\psi(s) v^{\prime}\right)\right)-\exp \left(-2 \pi i \eta \cdot \Upsilon_{\kappa-1}\left(\psi(s) v^{\prime}\right)\right)\right) \\
& \quad \times \Omega_{\mu}\left(u^{\prime}, v^{\prime}\right) d \sigma_{m}\left(u^{\prime}\right) d \sigma_{n}\left(v^{\prime}\right) h(r, s) \frac{d r d s}{r s} \mid \\
& =\left|\int_{d_{\mu}}^{a_{\mu}^{j+1}} \int_{a_{\mu}^{k}}^{a_{\mu}^{k+1}}\left(\widehat{\sigma_{\mu ; r, s}} \widehat{l, k}(\xi, \eta)-\widehat{\sigma_{\mu ; r, s}^{l, k-1}}(\xi, \eta)\right) h(r, s) \frac{d r d s}{r s}\right|
\end{aligned}
$$




$$
\begin{aligned}
& \leq C\|h\|_{\mu, \gamma}\left(\int_{d_{\mu}^{j}}^{a_{\mu}^{j+1}} \int_{a_{\mu}^{k}}^{a_{\mu}^{k+1}}\left|\widehat{\sigma_{\mu ; r, s}^{\widehat{l, k}}}(\xi, \eta)-\widehat{\sigma_{\mu ;, s}^{l, k-1}}(\xi, \eta)\right|^{\gamma^{\prime}} \frac{d r d s}{r s}\right)^{1 / \gamma^{\prime}} \\
& \leq C\|h\|_{\mu, \gamma}(\mu+1)^{2 \max \left\{1 / \gamma^{\prime}-1 / 2,0\right\}}\left(\int_{d_{\mu}^{j}}^{d_{\mu}^{j+1}} \int_{a_{\mu}^{k}}^{a_{\mu}^{k+1}}\left|\widehat{\sigma_{\mu ; r, s}^{\widehat{l, k}}}(\xi, \eta)-\widehat{\sigma_{\mu ; r, s}^{l, k-1}}(\xi, \eta)\right|^{2} \frac{d r d s}{r s}\right)^{1 / \tilde{\gamma}} .
\end{aligned}
$$

Combining this inequality with (2.11) implies (2.28). Applying similar arguments as in getting (2.28) and using (2.12), we get (2.29). By a change of variables and Hölder's inequality,

$$
\begin{aligned}
& \left|\widehat{\tau_{\mu ; k, j}^{\imath, \kappa}}(\xi, \eta)-\widehat{\tau_{\mu ; k, j}^{l-1, k}}(\xi, \eta)-\widehat{\tau_{\mu ; k, j}^{l, k-1}}(\xi, \eta)+\widehat{\tau_{\mu ; k, j}^{l-1, \kappa-1}}(\xi, \eta)\right| \\
& =\int_{d_{\mu}^{j}}^{d_{\mu}^{j+1}} \int_{a_{\mu}^{k}}^{a_{\mu}^{k+1}}\left(\widehat{\sigma_{\mu ; r, s}(\xi,}(\xi, \eta)-\widehat{\sigma_{\mu ; r, s}^{l-1, \kappa}}(\xi, \eta)-\widehat{\sigma_{\mu ; r, s}^{l, k-1}}(\xi, \eta)+\widehat{\sigma_{\mu ; r, s}^{l-1, k-1}}(\xi, \eta)\right) h(r, s) \frac{d r d s}{r s} \\
& \leq C\|h\|_{\mu, \gamma}\left(\int_{a_{\mu}^{j}}^{a_{\mu}^{j+1}} \int_{a_{\mu}^{k}}^{a_{\mu}^{k+1}} \mid \widehat{\sigma_{\mu ; r, s}^{l, k}}(\xi, \eta)-\widehat{\sigma_{\mu ; r, s}^{l-1, k}}(\xi, \eta)\right. \\
& \left.-\widehat{\sigma_{\mu ; r, s}^{l, k-1}}(\xi, \eta)+\left.\widehat{\sigma_{\mu ; r, s}^{l i, k-1}}(\xi, \eta)\right|^{\gamma^{\prime}} \frac{d r d s}{r s}\right)^{1 / \gamma^{\prime}} \\
& \leq C\|h\|_{\mu, \gamma}(\mu+1)^{2 \max \left\{1 / \gamma^{\prime}-1 / 2,0\right\}} \\
& \times\left(\int_{d_{\mu}^{j}}^{d_{\mu}^{j+1}} \int_{a_{\mu}^{k}}^{a_{\mu}^{k+1}}\left|\widehat{\sigma_{\mu ; r, r, s}^{l, k}}(\xi, \eta)-\widehat{\sigma_{\mu ;, r, s}^{l-1, \kappa}}(\xi, \eta)-\widehat{\sigma_{\mu ; r, s}^{l, k-1}}(\xi, \eta)+\widehat{\sigma_{\mu ; r, s}^{l-1, \kappa-1}}(\xi, \eta)\right|^{2} \frac{d r d s}{r s}\right)^{1 / \tilde{\gamma}} .
\end{aligned}
$$

This inequality, together with (2.13), yields (2.30) and finishes the proof.

Lemma 2.6 ([22]) Let $\mathcal{P}$ be a polynomial mapping: $\mathbb{R}_{+} \rightarrow \mathbb{R}^{n}$, where $\mathcal{P}(t)=\left(P_{1}(t), \ldots\right.$, $\left.P_{n}(t)\right)$ and $P_{i}(i=1,2, \ldots, n)$ are real polynomials defined on $\mathbb{R}_{+}$. Suppose $\gamma>1$ and $\phi \in \mathfrak{F}_{1}$ or $\mathfrak{F}_{2}$. Then the operator $M_{\phi}$ defined by

$$
M_{\phi}(f)(x)=\sup _{\kappa \in \mathbb{Z}} \int_{2^{\gamma \kappa}}^{2^{\gamma(\kappa+1)}}|f(x-\mathcal{P}(\phi(t)))| \frac{d t}{t}
$$

satisfies

$$
\left\|M_{\phi}(f)\right\|_{p} \leq C \gamma\|f\|_{p}
$$

for $1<p<\infty$. The constant $C$ is independent of $\gamma$ and the coefficients of $P_{i}(i=1,2, \ldots, n)$ but depends on $\phi$.

Applying Lemma 2.6, we have the following:

Lemma 2.7 Let $\mu \in \mathcal{D} \cup\{0\}$ and $\|h\|_{\mu, \gamma}<\infty$ for some $\gamma>1$. Suppose $\varphi, \psi \in \mathfrak{F}_{1}$ or $\mathfrak{F}_{2}$. Then, for $\iota \in\left\{0,1, \ldots, \lambda_{1}\right\}$ and $\kappa \in\left\{0,1, \ldots, \lambda_{2}\right\}$, the operator $\tau_{\mu: \iota, \kappa}^{*}$ satisfies

$$
\left\|\tau_{\mu:,, \kappa}^{*}(f)\right\|_{p} \leq C\|h\|_{\mu, \gamma}(\mu+1)^{2 / \gamma^{\prime}}\|f\|_{p} \quad \text { for } \gamma^{\prime}<p \leq \infty,
$$

where

$$
\tau_{\mu ; l, \kappa}^{*}(f)(x, y)=\sup _{k, j \in \mathbb{Z}}|| \tau_{\mu ; k, j}^{\imath, \kappa}|* f(x, y)|
$$


Proof We define the measures $\left\{\left|\Lambda_{\mu ; k, j}^{\iota, \kappa}\right|\right\}$ and the maximal operator $\Lambda_{\mu ; \iota, \kappa}^{*}$ by

$$
\widehat{\left|\Lambda_{\mu ; k, j}^{l, k}\right|}(\xi, \eta)=\iint_{\Delta_{\mu ; k, j}} \exp \left(-2 \pi i\left(\xi \cdot \Gamma_{\iota}\left(\varphi(|u|) u^{\prime}\right)+\eta \cdot \Upsilon_{\kappa}\left(\psi(|v|) v^{\prime}\right)\right)\right) \frac{\left|\Omega_{\mu}\left(u^{\prime}, v^{\prime}\right)\right|}{|u|^{m}|v|^{n}} d u d v
$$

and

$$
\Lambda_{\mu ; \iota, \kappa}^{*}(f)(x, y)=\sup _{k, j \in \mathbb{Z}}|| \Lambda_{\mu ; k, j}^{\iota, k}|* f(x, y)|
$$

where $\Delta_{\mu ; k, j}$ is defied as in (2.26). By a change of variables we have

$$
\begin{aligned}
& \Lambda_{\mu ; \iota, \kappa}^{*}(f)(x, y) \\
& =\sup _{k, j \in \mathbb{Z}}\left|\iint_{\Delta_{\mu ; k, j}} f\left(x-\Gamma_{\iota}\left(\varphi(|u|) u^{\prime}\right), y-\Upsilon_{\kappa}\left(\psi(|v|) v^{\prime}\right)\right) \frac{\left|\Omega_{\mu}\left(u^{\prime}, v^{\prime}\right)\right|}{|u|^{m}|v|^{n}} d u d v\right| \\
& \leq \iint_{S^{m-1} \times S^{n-1}} \sup _{k, j \in \mathbb{Z}} \int_{d_{\mu}^{j}}^{d_{\mu}^{j+1}} \int_{a_{\mu}^{k}}^{a_{\mu}^{k+1}}\left|f\left(x-\Gamma_{\iota}\left(\varphi(r) u^{\prime}\right), y-\Upsilon_{\kappa}\left(\psi(s) v^{\prime}\right)\right)\right| \frac{d r d s}{r s} \\
& \quad \times\left|\Omega_{\mu}\left(u^{\prime}, v^{\prime}\right)\right| d \sigma_{m}\left(u^{\prime}\right) d \sigma_{n}\left(v^{\prime}\right) .
\end{aligned}
$$

By the definition of $\Phi_{\iota}$ and $\Psi_{\kappa}$, using the iterated integration, Lemma 2.5, and Minkowski's inequality, we obtain

$$
\left\|\Lambda_{\mu ;, L}^{*}(f)\right\|_{p} \leq C(\mu+1)^{2}\|f\|_{p}, \quad 1<p<\infty .
$$

By a change of variables and Hölder's inequality we have

$$
\begin{aligned}
& || \tau_{\mu ; k, j}^{\ell, \kappa}|* f(x, y)| \\
& =\left|\iint_{\Delta_{\mu ; k, j}} f\left(x-\Gamma_{\iota}\left(\varphi(r) u^{\prime}\right), y-\Upsilon_{\kappa}\left(\psi(s) v^{\prime}\right)\right) \frac{\left|\Omega_{\mu}\left(u^{\prime}, v^{\prime}\right) h(|u|,|v|)\right|}{|u|^{m}|v|^{n}} d u d v\right| \\
& \leq \int_{d_{\mu}^{j}}^{a_{\mu}^{j+1}} \int_{a_{\mu}^{k}}^{a_{\mu}^{k+1}} \iint_{S^{m-1} \times S^{n-1}}\left|f\left(x-\Gamma_{\iota}\left(\varphi(r) u^{\prime}\right), y-\Upsilon_{\kappa}\left(\psi(s) v^{\prime}\right)\right)\right| \\
& \times\left|\Omega_{\mu}\left(u^{\prime}, v^{\prime}\right)\right| d \sigma_{m}\left(u^{\prime}\right) d \sigma_{n}\left(v^{\prime}\right)|h(r, s)| \frac{d r d s}{r s} \\
& \leq C\|h\|_{\mu, \gamma}\left(\int_{a_{\mu}^{j}}^{d_{\mu}^{j+1}} \int_{a_{\mu}^{k}}^{a_{\mu}^{k+1}}\left|\iint_{S^{m-1} \times S^{n-1}}\right| f\left(x-\Gamma_{\iota}\left(\varphi(r) u^{\prime}\right), y-\Upsilon_{\kappa}\left(\psi(s) v^{\prime}\right)\right) \mid\right. \\
& \left.\times\left.\left|\Omega_{\mu}\left(u^{\prime}, v^{\prime}\right)\right| d \sigma_{m}\left(u^{\prime}\right) d \sigma_{n}\left(v^{\prime}\right)\right|^{\gamma^{\prime}} \frac{d r d s}{r s}\right)^{1 / \gamma^{\prime}} \\
& \leq C\|h\|_{\mu, \gamma}\left(\int_{a_{\mu}^{j}}^{d_{\mu}^{j+1}} \int_{a_{\mu}^{k}}^{a_{\mu}^{k+1}} \iint_{S^{m-1} \times S^{n-1}}\left|f\left(x-\Gamma_{\iota}\left(\varphi(r) u^{\prime}\right), y-\Upsilon_{\kappa}\left(\psi(s) v^{\prime}\right)\right)\right|^{\gamma^{\prime}}\right. \\
& \left.\times\left|\Omega_{\mu}\left(u^{\prime}, v^{\prime}\right)\right| d \sigma_{m}\left(u^{\prime}\right) d \sigma_{n}\left(v^{\prime}\right) \frac{d r d s}{r s}\right)^{1 / \gamma^{\prime}} \\
& \leq C\|h\|_{\mu, \gamma}\left(\Lambda_{\mu ; \iota, \kappa}^{*}\left(|f|^{\gamma^{\prime}}\right)(x, y)\right)^{1 / \gamma^{\prime}} \text {. }
\end{aligned}
$$

Combining this inequality with (2.32) yields (2.31). 
For $\iota \in\left\{1, \ldots, \lambda_{1}\right\}$ and $\kappa \in\left\{1, \ldots, \lambda_{2}\right\}$, let $r_{1, \iota}=\operatorname{rank}\left(\mathcal{L}_{\iota}\right)$ and $r_{2, \iota}=\operatorname{rank}\left(\Theta_{\kappa}\right)$. By a change of basic matrices (see also [21], Lemma 6.1), there are four nonsingular linear transformations $\mathcal{H}_{1, \iota}: \mathbb{R}^{r_{1, \iota}} \rightarrow \mathbb{R}^{r_{1, \iota}}, \mathcal{H}_{2, \iota}: \mathbb{R}^{r_{2, \kappa}} \rightarrow \mathbb{R}^{r_{2, \kappa}}, \mathcal{G}_{1, l}: \mathbb{R}^{M} \rightarrow \mathbb{R}^{M}$, and $\mathcal{G}_{2, \kappa}: \mathbb{R}^{N} \rightarrow \mathbb{R}^{N}$ such that

$$
\begin{aligned}
& \left|\mathcal{H}_{1, \iota} \pi_{r_{1, \iota}}^{M} \mathcal{G}_{1, l} \xi\right| \leq\left|\mathcal{L}_{\iota}(\xi)\right| \leq C_{M}\left|\mathcal{H}_{1, \iota} \pi_{r_{1, l}}^{M} \mathcal{G}_{1, l} \xi\right| \quad \text { for } \xi \in \mathbb{R}^{M}, \\
& \left|\mathcal{H}_{2, \kappa} \pi_{r_{2, \kappa}}^{N} \mathcal{G}_{2, \kappa} \eta\right| \leq\left|\Theta_{\kappa}(\eta)\right| \leq C_{N}\left|\mathcal{H}_{2, \kappa} \pi_{r_{2, \kappa}}^{N} \mathcal{G}_{2, \kappa} \eta\right| \quad \text { for } \xi \in \mathbb{R}^{N},
\end{aligned}
$$

where $C_{M}, C_{N}>1, \pi_{r_{1, l}}^{M}$ is a projection operator from $\mathbb{R}^{M}$ to $\mathbb{R}^{r_{1, l}}$, and $\pi_{r_{1, l}}^{N}$ is the projection operator from $\mathbb{R}^{N}$ to $\mathbb{R}^{r_{2, \kappa}}$.

Now we take two radial functions $\phi_{1}, \phi_{2} \in C_{0}^{\infty}(\mathbb{R})$ such that $\phi_{1}(t)=\phi_{2}(s) \equiv 1$ for $\max \{|t|,|s|\} \leq 1$ and $\phi_{1}(t)=\phi_{2}(s) \equiv 0$ for $\min \{|t|,|s|\}>a_{\mu}$. For $\iota \in\left\{1, \ldots, \lambda_{1}\right\}$ and $\kappa \in$ $\left\{1, \ldots, \lambda_{2}\right\}$, we define the measures $\left\{\omega_{\mu ; r, s}^{\iota, \kappa}\right\}$ and $\left\{\lambda_{\mu ; k, j}^{\ell, \kappa}\right\}$ by

$$
\begin{aligned}
\widehat{\omega_{\mu ; r, s}^{\iota, \kappa}}(\xi, \eta)= & \widehat{\sigma_{\mu ; r, s}^{\iota, \kappa}}(\xi, \eta) \Pi_{1}(\iota) \Pi_{2}(\kappa)-\widehat{\sigma_{\mu ; r, s}^{l-1, \kappa}}(\xi, \eta) \Pi_{1}(\iota-1) \Pi_{2}(\kappa) \\
& -\widehat{\sigma_{\mu ; r, s}^{\iota, \kappa-1}}(\xi, \eta) \Pi_{1}(\iota) \Pi_{2}(\kappa-1)+\widehat{\sigma_{\mu ; r, s}^{l-1, \kappa-1}}(\xi, \eta) \Pi_{1}(\iota-1) \Pi_{2}(\kappa-1)
\end{aligned}
$$

and

$$
\begin{aligned}
\widehat{\lambda_{\mu ; k, j}^{\widehat{l, k}}}(\xi, \eta)= & \widehat{\tau_{\mu ; k, j}^{\imath, \kappa}}(\xi, \eta) \Pi_{1}(\iota) \Pi_{2}(\kappa)-\widehat{\tau_{\mu ; k, j}^{\imath-1, \kappa}}(\xi, \eta) \Pi_{1}(\iota-1) \Pi_{2}(\kappa) \\
& -\widehat{\tau_{\mu ; k, j}^{l, k-1}}(\xi, \eta) \Pi_{1}(\iota) \Pi_{2}(\kappa-1)+\widehat{\tau_{\mu ; k, j}^{l-1, \kappa-1}}(\xi, \eta) \Pi_{1}(\iota-1) \Pi_{2}(\kappa-1),
\end{aligned}
$$

where

$$
\begin{aligned}
& \Pi_{1}(\iota)=\prod_{i=l+1}^{\lambda_{1}} \phi_{1}\left(\left|\varphi\left(a_{\mu}^{k}\right)^{d_{\alpha_{i}}} \mathcal{H}_{1, i} \pi_{r_{1, i}}^{M} \mathcal{G}_{1, i} \xi\right|\right), \\
& \Pi_{2}(\kappa)=\prod_{\varsigma=\kappa+1}^{\lambda_{2}} \phi_{2}\left(\left|\psi\left(a_{\mu}^{j}\right)^{v_{\beta_{\zeta}}} \mathcal{H}_{2, \kappa} \pi_{r_{2, \kappa}}^{N} \mathcal{G}_{2, \kappa} \eta\right|\right) .
\end{aligned}
$$

Let $\left\{||_{\mu ; r, s}^{\ell, \kappa} \mid\right\}$ be defined in the same way as $\left\{\omega_{\mu ; r, s}^{\iota, \kappa}\right\}$, but with $\left\{\sigma_{\mu ; r, s}^{\ell, \kappa}\right\}$ replaced by $\left\{\left|\sigma_{\mu ; r, s}^{\ell, \kappa}\right|\right\}$. It is easy to see that

$$
\sigma_{\mu ; r, s}^{\lambda_{1}, \lambda_{2}}=\sum_{\iota=1}^{\lambda_{1}} \sum_{\kappa=1}^{\lambda_{2}} \omega_{\mu ;, r, s}^{\iota, \kappa}
$$

and

$$
\tau_{\mu ; k, j}^{\lambda_{1}, \lambda_{2}}=\sum_{l=1}^{\lambda_{1}} \sum_{\kappa=1}^{\lambda_{2}} \lambda_{\mu ; k, j}^{\iota, \kappa}
$$

Applying Lemmas 2.4 and 2.5 and combining with the arguments similar to those in the proof of [10], Lemma 2.7, we can obtain the following: 
Lemma 2.8 Let $\varphi, \psi \in \mathfrak{F}_{1}$ or $\mathfrak{F}_{2}$. For $\mu \in \mathcal{D} \cup\{0\}, k, j \in \mathbb{Z}, 1 \leq \iota \leq \lambda_{1}, 1 \leq \kappa \leq \lambda_{2}$, and $(\xi, \eta) \in \mathbb{R}^{M} \times \mathbb{R}^{N}$, there exist positive constants $\epsilon_{1}, \epsilon_{2}$, and $C$ such that

$$
\begin{aligned}
& \sup _{r, s>0}\left\|\omega_{\mu ; r, s}^{\ell, \kappa}\right\| \leq C \\
& \int_{a_{\mu}^{j}}^{d_{\mu}^{j+1}} \int_{a_{\mu}^{k}}^{a_{\mu}^{k+1}}\left|\widehat{\omega_{\mu ; r, s}^{l, k}}(\xi, \eta)\right|^{2} \frac{d r d s}{r s} \\
& \leq C(\mu+1)^{2} \min \left\{1,\left|\varphi\left(a_{\mu}^{k}\right)^{d_{\alpha_{l}}} \mathcal{L}_{\iota}(\xi)\right|^{\frac{1}{\mu+1}}\left|\psi\left(a_{\mu}^{j}\right)^{v_{\beta_{\kappa}}} \Theta_{\kappa}(\eta)\right|^{\frac{1}{\mu+1}},\right. \\
& \left|\varphi\left(a_{\mu}^{k}\right)^{d_{\alpha_{l}}} \mathcal{L}_{l}(\xi)\right|^{\frac{1}{\mu+1}}\left|\psi\left(a_{\mu}^{j}\right)^{v_{\beta_{\kappa}}} \Theta_{\kappa}(\eta)\right|^{-\frac{\epsilon_{2}}{\mu+1}}, \\
& \left|\psi\left(a_{\mu}^{j}\right)^{v_{\beta_{\kappa}}} \Theta_{\kappa}(\eta)\right|^{\frac{1}{\mu+1}}\left|\varphi\left(a_{\mu}^{k}\right)^{d_{\alpha_{l}}} \mathcal{L}_{\iota}(\xi)\right|^{-\frac{\epsilon_{1}}{\mu+1}}, \\
& \left.\left|\varphi\left(a_{\mu}^{k}\right)^{d_{\alpha_{l}}} \mathcal{L}_{\iota}(\xi)\right|^{-\frac{\epsilon_{1}}{\mu+1}}\left|\psi\left(a_{\mu}^{j}\right)^{v_{\beta_{\kappa}}} \Theta_{\kappa}(\eta)\right|^{-\frac{\epsilon_{2}}{\mu+1}}\right\} .
\end{aligned}
$$

Lemma 2.9 Let $\mu \in \mathcal{D} \cup\{0\}$ and $\|h\|_{\mu, \gamma}<\infty$ for some $\gamma>1$ and $\tilde{\gamma}=\max \left\{2, \gamma^{\prime}\right\}$. Suppose that $\varphi, \psi \in \mathfrak{F}_{1}$ or $\mathfrak{F}_{2}$. Then for $k, j \in \mathbb{Z}$, there exist positive constants $\epsilon_{1}, \epsilon_{2}, C$ such that, for any $1 \leq \iota \leq \lambda_{1}$ and $1 \leq \kappa \leq \lambda_{2}$,

$$
\begin{aligned}
& \sup _{k, j, \in \mathbb{Z}}\left\|\lambda_{\mu ; k, j}^{\iota, \kappa}\right\| \leq C\|h\|_{\mu, \gamma}(\mu+1)^{2 / \gamma^{\prime}} ; \\
& \left|\widehat{\lambda_{\mu ; k, j}^{\widehat{\lambda}}}(\xi, \eta)\right| \leq C\|h\|_{\mu, \gamma}(\mu+1)^{2 / \gamma^{\prime}} \min \left\{1,\left|\varphi\left(a_{\mu}^{k}\right)^{d_{\alpha_{l}}} \mathcal{L}_{l}(\xi)\right|^{\frac{1}{\hat{\gamma}(\mu+1)}}\left|\psi\left(a_{\mu}^{j}\right)^{v_{\beta_{K}}} \Theta_{\kappa}(\eta)\right|^{\frac{1}{\hat{\gamma}(\mu+1)}},\right. \\
& \left|\varphi\left(a_{\mu}^{k}\right)^{d_{\alpha_{l}}} \mathcal{L}_{l}(\xi)\right|^{\frac{1}{\bar{\gamma}(\mu+1)}}\left|\psi\left(a_{\mu}^{j}\right)^{v_{\beta_{\kappa}}} \Theta_{\kappa}(\eta)\right|^{-\frac{\epsilon_{2}}{\bar{\gamma}(\mu+1)}}, \\
& \left|\psi\left(a_{\mu}^{j}\right)^{v_{\beta_{\kappa}}} \Theta_{\kappa}(\eta)\right|^{\frac{1}{\bar{\gamma}(\mu+1)}}\left|\varphi\left(a_{\mu}^{k}\right)^{d_{\alpha_{l}}} \mathcal{L}_{l}(\xi)\right|^{-\frac{\epsilon_{1}}{\bar{\gamma}(\mu+1)}}, \\
& \left.\left|\varphi\left(a_{\mu}^{k}\right)^{d_{\alpha_{l}}} \mathcal{L}_{l}(\xi)\right|^{-\frac{\epsilon_{1}}{\tilde{\gamma}(\mu+1)}}\left|\psi\left(a_{\mu}^{j}\right)^{v_{\beta_{\kappa}}} \Theta_{\kappa}(\eta)\right|^{-\frac{\epsilon_{2}}{\gamma}(\mu+1)}\right\} .
\end{aligned}
$$

Applying Lemma 2.6 and the definition of $\lambda_{\mu ; k, j}^{l, k}$, we can establish the following:

Lemma 2.10 Let $\mu \in \mathcal{D} \cup\{0\}$ and $\|h\|_{\mu, \gamma}<\infty$ for some $\gamma>1$. Suppose that $\varphi, \psi \in \mathfrak{F}_{1}$ or $\mathfrak{F}_{2}$. Then, for $\iota \in\left\{0,1, \ldots, \lambda_{1}\right\}$ and $\kappa \in\left\{0,1, \ldots, \lambda_{2}\right\}$, there exists a constant $C>0$ such that

$$
\left\|\sup _{k, j \in \mathbb{Z}}|| \lambda_{\mu ; k, j}^{\iota, \kappa}|* f|\right\|_{p} \leq C\|h\|_{\mu, \gamma}(\mu+1)^{2 / \gamma^{\prime}}\|f\|_{p}, \quad \gamma^{\prime}<p \leq \infty
$$

Applying Lemma 2.10, by arguments similar to those used in the proof of [21], Theorem 7.5, we have the following:

Lemma 2.11 Let $\mu \in \mathcal{D} \cup\{0\}$ and $\|h\|_{\mu, \gamma}<\infty$ for some $\gamma>1$. Suppose that $\varphi, \psi \in \mathfrak{F}_{1}$ or $\mathfrak{F}_{2}$. Then, for $\iota \in\left\{0,1, \ldots, \lambda_{1}\right\}$ and $\kappa \in\left\{0,1, \ldots, \lambda_{2}\right\}$, there exists a constant $C>0$ such that

$$
\left\|\left(\sum_{k, j \in \mathbb{Z}}\left|\lambda_{\mu ; k, j}^{\ell, \kappa} * g_{k, j}\right|^{2}\right)^{1 / 2}\right\|_{p} \leq C\|h\|_{\mu, \gamma}(\mu+1)^{2 / \gamma^{\prime}}\left\|\left(\sum_{k, j \in \mathbb{Z}}\left|g_{k, j}\right|^{2}\right)^{1 / 2}\right\|_{p}
$$

for $p$ satisfying $|1 / p-1 / 2|<\min \left\{1 / \gamma^{\prime}, 1 / 2\right\}$. 
Lemma 2.12 Let $\Omega_{\mu}$ be as in (2.1) for $\mu \in \mathcal{D} \cup\{0\}$. Suppose that $\varphi, \psi \in \mathfrak{F}_{1}$ or $\mathfrak{F}_{2}$. For $\iota \in$ $\left\{1, \ldots, \lambda_{1}\right\}$ and $\kappa \in\left\{1, \ldots, \lambda_{2}\right\}$, define the operator $\mathcal{U}$ by

$$
\mathcal{U}(f)(x, y)=\sup _{k, j \in \mathbb{Z}} \int_{a_{\mu}^{j}}^{a_{\mu}^{j+1}} \int_{a_{\mu}^{k}}^{a_{\mu}^{k+1}}|| \omega_{\mu ;, s}^{\iota, \kappa}|* f(x, y)| \frac{d r d s}{r s} .
$$

Then there exists a constant $C>0$ such that

$$
\|\mathcal{U}(f)\|_{p} \leq C(\mu+1)^{2}\|f\|_{p}
$$

for all $f \in L^{p}\left(\mathbb{R}^{M} \times \mathbb{R}^{N}\right)$ and $1<p<\infty$.

Proof Define the operator $\mathcal{H}$ by

$$
\mathcal{H}(f)(x, y)=\sup _{k, j \in \mathbb{Z}} \int_{a_{\mu}^{j}}^{a_{\mu}^{j+1}} \int_{a_{\mu}^{k}}^{a_{\mu}^{k+1}}|| \sigma_{\mu ; r, s}^{\iota, \kappa}|* f(x, y)| \frac{d r d s}{r s} .
$$

Then

$$
\begin{aligned}
\mathcal{H}(f)(x, y)= & \sup _{k, j \in \mathbb{Z}} \int_{a_{\mu}^{j}}^{a_{\mu}^{j+1}} \int_{a_{\mu}^{k}}^{a_{\mu}^{k+1}} \mid \iint_{S^{m-1} \times S^{n-1}} f\left(x-\Phi_{\iota}\left(u^{\prime}\right), y-\Psi_{\kappa}\left(v^{\prime}\right)\right) \\
& \times\left|\Omega_{\mu}\left(u^{\prime}, v^{\prime}\right)\right| d \sigma\left(u^{\prime}\right) d \sigma\left(v^{\prime}\right) \mid \frac{d r d s}{r s} \\
\leq & C \iint_{S^{m-1} \times S^{n-1}} \sup _{k, j \in \mathbb{Z}} \int_{a_{\mu}^{j}}^{a_{\mu}^{j+1}} \int_{a_{\mu}^{k}}^{a_{\mu}^{k+1}}\left|f\left(x-\Phi_{\iota}\left(u^{\prime}\right), y-\Psi_{\kappa}\left(v^{\prime}\right)\right)\right| \frac{d r d s}{r s} \\
& \times\left|\Omega_{\mu}\left(u^{\prime}, v^{\prime}\right)\right| d \sigma\left(u^{\prime}\right) d \sigma\left(v^{\prime}\right) .
\end{aligned}
$$

Invoking Lemma 2.6, using the iterated integration and Minkowski's inequality, we can obtain

$$
\|\mathcal{H}(f)\|_{p} \leq C(\mu+1)^{2}\|f\|_{p}, \quad 1<p<\infty .
$$

This, together with the definition of $\omega_{\mu ; r, s}^{\iota, \kappa}$ implies Lemma 2.12.

\section{Proofs of main results}

Proof of Theorem 1.1 We only prove the case $\varphi, \psi \in \mathfrak{F}_{1}$, and the other cases are analogous. By Remark 1.1 there exist $B_{\varphi}, B_{\psi}>1$ such that $\varphi(2 t) \geq B_{\varphi} \varphi(t)$ and $\psi(2 t) \geq B_{\psi} \psi(t)$ for all $t>0$. It follows form (2.4) and (2.36) that

$$
\begin{aligned}
& T_{h, \Omega, \Gamma, \Upsilon}(f) \leq \sum_{\mu \in \mathcal{D} \cup\{0\}} \lambda_{\mu} T_{h, \Omega_{\mu}, \Gamma, \Upsilon}(f), \\
& T_{h, \Omega_{\mu}, \Gamma, \Upsilon}(f)=\sum_{k, j \in \mathbb{Z}} \tau_{\mu ; k, j}^{\lambda_{1}, \lambda_{2}} * f=\sum_{\imath=1}^{\lambda_{1}} \sum_{\kappa=1}^{\lambda_{2}} \sum_{k, j \in \mathbb{Z}} \lambda_{\mu ; k, j}^{\iota, \kappa} * f=: \sum_{\imath=1}^{\lambda_{1}} \sum_{\kappa=1}^{\lambda_{2}} T_{h, \Omega_{\mu}, \Gamma, \Upsilon}^{\iota, \kappa}(f) .
\end{aligned}
$$


Then by (2.5), (3.1), and (3.2), to prove Theorem 1.1, it suffices to show that, for any $\iota \in$ $\left\{1, \ldots, \lambda_{1}\right\}$ and $\kappa \in\left\{1, \ldots, \lambda_{2}\right\}$,

$$
\left\|T_{h, \Omega_{\mu}, \Gamma, \Upsilon}^{l, \kappa}(f)\right\|_{p} \leq C(\mu+1)^{2} \mid f \|_{p} \quad \text { for }|1 / p-1 / 2|<\min \left\{1 / \gamma^{\prime}, 1 / 2\right\}
$$

Without loss of generality, we may assume that $\left\{d_{\alpha_{l}}\right\}_{l=1}^{\lambda_{1}}$ and $\left\{v_{\beta_{\kappa}}\right\}_{\kappa=1}^{\lambda_{2}}$ are two sequences of positive numbers. For fixed $\iota \in\left\{1, \ldots, \lambda_{1}\right\}$ and $\kappa \in\left\{1, \ldots, \lambda_{2}\right\}$, we can choose two collections of $C^{\infty}$ functions $\left\{\zeta_{i}\right\}_{i \in \mathbb{Z}}$ and $\left\{\eta_{l}\right\}_{l \in \mathbb{Z}}$ on $(0, \infty)$ with the following properties:

(i) $\operatorname{supp}\left(\zeta_{i}\right) \subset\left[\varphi\left(a_{\mu}^{i+1}\right)^{-d_{\alpha_{l}}}, \varphi\left(a_{\mu}^{i-1}\right)^{-d_{\alpha_{l}}}\right], \operatorname{supp}\left(\eta_{l}\right) \subset\left[\psi\left(a_{\mu}^{l+1}\right)^{-v_{\beta_{\kappa}}}, \psi\left(a_{\mu}^{l-1}\right)^{-v_{\beta_{\kappa}}}\right]$;

(ii) $0 \leq \zeta_{i}, \eta_{l} \leq 1, \sum_{i \in \mathbb{Z}} \zeta_{i}(t)^{2}=\sum_{l \in \mathbb{Z}} \eta_{l}(t)^{2}=1$;

(iii) $\left|(d / d t)^{\varsigma} \zeta_{i}(t)\right| \leq C_{1} / t,\left|(d / d t)^{\varsigma} \eta_{l}(t)\right| \leq C_{2} / t$, where $C_{1}, C_{2}$ are independent of $i, l, \varsigma$.

Define the multiplier operator $S_{i, l}$ on $\mathbb{R}^{M} \times \mathbb{R}^{N}$ by

$$
\widehat{S_{i, l} f}(x, y)=\zeta_{i}\left(\left|\mathcal{H}_{1, l} \pi_{r_{1, l}}^{M} \mathcal{G}_{1, l} x\right|\right) \eta_{l}\left(\left|\mathcal{H}_{2, \kappa} \pi_{r_{2, \kappa}}^{N} \mathcal{G}_{2, \kappa} y\right|\right) \hat{f}(x, y)
$$

We can write

$$
T_{h, \Omega_{\mu}, \Gamma, \Upsilon}^{\iota, \kappa}(f)=\sum_{i, l \in \mathbb{Z}} \sum_{k, j \in \mathbb{Z}} S_{i+k, l+j}\left(\lambda_{k, j \mu}^{\iota, \kappa} * S_{i+k, l+j} f\right)=: \sum_{i, l \in \mathbb{Z}} T_{i, l} f
$$

Now we consider the $L^{p}$-boundedness of $T_{i, l}$. Applying the Littlewood-Paley theory and Lemma 2.11, we have that, for any $p$ satisfying $|1 / p-1 / 2|<\min \left\{1 / \gamma^{\prime}, 1 / 2\right\}$,

$$
\begin{aligned}
\left\|T_{i, l} f\right\|_{p} & \leq C\left\|\left(\sum_{k, j \in \mathbb{Z}}\left|S_{i+k, l+j}\left(\lambda_{\mu ; k, j}^{\iota, k} * S_{i+k, l+j} f\right)\right|^{2}\right)^{1 / 2}\right\|_{p} \\
& \leq C\left\|\left(\sum_{k, j \in \mathbb{Z}}\left|\lambda_{\mu ; k, j}^{l, k} * S_{i+k, l+j} f\right|^{2}\right)^{1 / 2}\right\|_{p} \\
& \leq C\|h\|_{\mu, \gamma}(\mu+1)^{2 / \gamma^{\prime}}\left\|\left(\sum_{k, j \in \mathbb{Z}} \mid S_{i+k, l+f} f^{2}\right)^{1 / 2}\right\|_{p} \\
& \leq C\|h\|_{\mu, \gamma}(\mu+1)^{2 / \gamma^{\prime}}\|f\|_{p} .
\end{aligned}
$$

On the other hand, by the Littlewood-Paley theory and Plancherel's theorem we have

$$
\begin{aligned}
& \left\|T_{i, l} f\right\|_{2}^{2} \leq C\left\|\left(\sum_{k, j \in \mathbb{Z}}\left|\lambda_{\mu ; k, j}^{l, k} * S_{i+k, l+j} f\right|^{2}\right)^{1 / 2}\right\|_{2}^{2} \\
& =C \sum_{k, j \in \mathbb{Z}} \iint_{\mathbb{R}^{M} \times \mathbb{R}^{N}}\left|\widehat{\lambda_{\mu ; k, j}^{\widehat{\imath},}}(\xi, \eta)\right|^{2} \mid \zeta_{i+k}\left(\left|\mathcal{H}_{1, l} \pi_{r_{1, l}}^{M} \mathcal{G}_{1, l} x\right|\right) \\
& \times\left.\eta_{l+j}\left(\left|\mathcal{H}_{2, \kappa} \pi_{r_{2, \kappa}}^{N} \mathcal{G}_{2, \kappa} y\right|\right)\right|^{2}|\hat{f}(\xi, \eta)|^{2} d \xi d \eta \\
& \leq C \sum_{k, j \in \mathbb{Z}} \iint_{E_{i+k, l+j}}\left|\widehat{\lambda_{\mu ; k, j} \widehat{, k}}(\xi, \eta)\right|^{2}|\hat{f}(\xi, \eta)|^{2} d \xi d \eta,
\end{aligned}
$$


where

$$
\begin{aligned}
E_{i+k, l+j}= & \left\{(\xi, \eta) \in \mathbb{R}^{M} \times \mathbb{R}^{N}: \varphi\left(a_{\mu}^{i+k+1}\right)^{-d_{\alpha_{\iota}}} \leq\left|\mathcal{H}_{1, l} \pi_{r_{1, l}}^{M} \mathcal{G}_{1, \iota} \xi\right| \leq \varphi\left(a_{\mu}^{i+k-1}\right)^{-d_{\alpha_{\ell}}},\right. \\
& \left.\psi\left(a_{\mu}^{l+j+1}\right)^{-v_{\beta_{\kappa}}} \leq\left|\mathcal{H}_{2, \kappa} \pi_{r_{2, \kappa}}^{N} \mathcal{G}_{2, \kappa} \eta\right| \leq \psi\left(a_{\mu}^{l+j-1}\right)^{-v_{\beta_{\kappa}}}\right\} .
\end{aligned}
$$

Using Lemma 2.9 and (2.33)-(2.34), we have

$$
\left\|T_{i, l} f\right\|_{2} \leq C\|h\|_{\mu, \gamma}(\mu+1)^{2 / \gamma^{\prime}} B_{i, l}\|f\|_{2}
$$

where

$$
B_{i, l}= \begin{cases}B_{\varphi}^{-i d_{\alpha_{l}} l \tilde{\gamma}} B_{\psi}^{-l v_{\beta_{k}} / \tilde{\gamma}}, & i, l>-1, \\ B_{\varphi}^{-i d_{\alpha_{l}} l \tilde{\gamma}} B_{\psi}^{l \epsilon_{2} v_{\beta_{\kappa}} / \tilde{\gamma}}, & i>-1, l \leq-1, \\ B_{\varphi}^{i \epsilon_{1} d_{\alpha_{l}} / \tilde{\gamma}} B_{\psi}^{-l v_{\beta_{\kappa}} / \tilde{\gamma}}, & i \leq-1, l>-1, \\ B_{\varphi}^{i \epsilon_{1} d_{\alpha_{l}} / \tilde{\gamma}} B_{\psi}^{l \epsilon_{2} v_{\beta_{\kappa}} / \tilde{\gamma}}, & i, l \leq-1 .\end{cases}
$$

Here $\tilde{\gamma}=\max \left\{2, \gamma^{\prime}\right\}$. Interpolating (3.5) and (3.7), for any $p$ satisfying $|1 / p-1 / 2|<$ $\min \left\{1 / \gamma^{\prime}, 1 / 2\right\}$, there exists $\delta \in(0,1]$ such that

$$
\left\|T_{i, f} f\right\|_{p} \leq C\|h\|_{\mu, \gamma}(\mu+1)^{2 / \gamma^{\prime}} B_{i, l}^{\delta}\|f\|_{p}, \quad|1 / p-1 / 2|<\min \left\{1 / \gamma^{\prime}, 1 / 2\right\}
$$

Then we have for any $p$ satisfying $|1 / p-1 / 2|<\min \left\{1 / \gamma^{\prime}, 1 / 2\right\}$,

$$
\begin{aligned}
\sum_{i, l \in \mathbb{Z}}\left\|T_{i, l} f\right\|_{p} \leq & C\|h\|_{\mu, \gamma}(\mu+1)^{2 / \gamma^{\prime}}\left(\sum_{i, l>-1} B_{\varphi}^{-i d_{\alpha_{\ell}} \delta \tilde{\gamma}} B_{\psi}^{-l v_{\beta_{\kappa}} \delta / \tilde{\gamma}}+\sum_{i>-1, l \leq-1} B_{\varphi}^{-i d d_{\alpha_{l}} \delta / \tilde{\gamma}} B_{\psi}^{l \epsilon_{2} v_{\beta_{\kappa}} \delta / \tilde{\gamma}}\right. \\
& \left.+\sum_{i \leq-1, l>-1} B_{\varphi}^{i \epsilon_{1} d_{\alpha_{l}} \delta / \tilde{\gamma}} B_{\psi}^{-l v_{\beta_{\kappa}} \delta / \tilde{\gamma}}+\sum_{i, l \leq-1} B_{\varphi}^{i \epsilon_{1} d_{\alpha_{l}} \delta / \tilde{\gamma}} B_{\psi}^{l \epsilon_{2} v_{\beta_{\kappa}} \delta / \tilde{\gamma}}\right)\|f\|_{p} \\
\leq & C\|h\|_{\mu, \gamma}(\mu+1)^{2 / \gamma^{\prime}}\|f\|_{p} .
\end{aligned}
$$

This, combined with (3.4) and Minkowski's inequality, implies that, for any $\iota \in\left\{1, \ldots, \lambda_{1}\right\}$ and $\kappa \in\left\{1, \ldots, \lambda_{2}\right\}$,

$$
\left\|T_{h, \Omega_{\mu}, \Gamma, \Upsilon}^{l, \kappa}(f)\right\|_{p} \leq C\|h\|_{\mu, \gamma}(\mu+1)^{2 / \gamma^{\prime}}\|f\|_{p} \quad \text { for }|1 / p-1 / 2|<\min \left\{1 / \gamma^{\prime}, 1 / 2\right\} .
$$

Estimate (3.3) follows from (3.9) and the fact $\|h\|_{\mu, \gamma} \leq(\mu+1)^{2 / \gamma}\|h\|_{\Delta_{\gamma}}$. This proves Theorem 1.1.

Proof of Theorem 1.3 We only prove the case $\varphi, \psi \in \mathfrak{F}_{1}$, and the other cases are analogous. It follows from (2.4) that

$$
M_{\Omega, \Gamma, \Upsilon}^{(\gamma)}(f) \leq \sum_{\mu \in D \cup\{0\}} \lambda_{\mu} M_{\Omega_{\mu}, \Gamma, \Upsilon}^{(\gamma)}(f)
$$

Hence, by (2.5), to prove Theorem 1.3, it suffices to show that

$$
\left\|M_{\Omega_{\mu}, \Gamma, \Upsilon}^{(\gamma)}(f)\right\|_{p} \leq C(\mu+1)^{2 / \gamma^{\prime}}\|f\|_{p}
$$


for $\gamma^{\prime} \leq p<\infty$ if $1 \leq \gamma \leq 2$ and for $p=\infty$ if $\gamma=1$. By duality, Hölder's inequality, Minkowski's inequality, and (2.35) we have

$$
\begin{aligned}
M_{\Omega_{\mu}, \Gamma, \Upsilon}^{(\gamma)}(f)(x, y) & =\sup _{\|h\|_{U_{\gamma}} \leq 1}\left|\int_{0}^{\infty} \int_{0}^{\infty} f * \sigma_{\mu ;, r, s}^{\lambda_{1}, \lambda_{2}}(x, y) h(r, s) \frac{d r d s}{r s}\right| \\
& \leq\left(\int_{0}^{\infty} \int_{0}^{\infty}\left|f * \sigma_{\mu ; r, s}^{\lambda_{1}, \lambda_{2}}(x, y)\right|^{\gamma^{\prime}} \frac{d r d s}{r s}\right)^{1 / \gamma^{\prime}} \\
& =\left(\int_{0}^{\infty} \int_{0}^{\infty}\left|f * \sum_{\imath=1}^{\lambda_{1}} \sum_{\kappa=1}^{\lambda_{2}} \omega_{\mu ;, r, s}^{\iota, \kappa}(x, y)\right|^{\gamma^{\prime}} \frac{d r d s}{r s}\right)^{1 / \gamma^{\prime}} \\
& \leq \sum_{\iota=1}^{\lambda_{1}} \sum_{\kappa=1}^{\lambda_{2}}\left(\int_{0}^{\infty} \int_{0}^{\infty}\left|f * \omega_{\mu ;, r, s}^{\iota \iota, \kappa}(x, y)\right|^{\gamma^{\prime}} \frac{d r d s}{r s}\right)^{1 / \gamma^{\prime}} \\
& :=\sum_{l=1}^{\lambda_{1}} \sum_{\kappa=1}^{\lambda_{2}} M_{l, \kappa}^{\left(\gamma^{\prime}\right)}(f)(x, y) .
\end{aligned}
$$

We shall consider the following three cases.

Case $1(\gamma=2)$. By Minkowski's inequality we have

$$
\begin{aligned}
M_{l, k}^{(2)}(f)(x, y) & =\left(\int_{0}^{\infty} \int_{0}^{\infty}\left|f * \omega_{\mu ; r, s}^{\iota, \kappa}(x, y)\right|^{2} \frac{d r d s}{r s}\right)^{1 / 2} \\
& =\left(\sum_{k, j \in \mathbb{Z}} \int_{a_{\mu}^{j}}^{d_{\mu}^{j+1}} \int_{a_{\mu}^{k}}^{a_{\mu}^{k+1}}\left|f * \omega_{\mu ; r, s}^{\iota, \kappa}(x, y)\right|^{2} \frac{d r d s}{r s}\right)^{1 / 2} \\
& =\left(\sum_{k, j \in \mathbb{Z}} \int_{a_{\mu}^{j}}^{d_{\mu}^{j+1}} \int_{a_{\mu}^{k}}^{a_{\mu}^{k+1}}\left|\omega_{\mu ; r, s}^{\iota, \kappa} *\left(\sum_{i, l \in \mathbb{Z}} S_{i+k, l+j} S_{i+k, l+j} f\right)(x, y)\right|^{2} \frac{d r d s}{r s}\right)^{1 / 2} \\
& \leq \sum_{i, l \in \mathbb{Z}}\left(\sum_{k, j \in \mathbb{Z}} \int_{d_{\mu}^{j}}^{a_{\mu}^{j+1}} \int_{a_{\mu}^{k}}^{a_{\mu}^{k+1}}\left|\omega_{\mu ; r, s}^{\iota, \kappa} *\left(S_{i+k, l+j} S_{i+k, l+j} f\right)(x, y)\right|^{2} \frac{d r d s}{r s}\right)^{1 / 2} \\
& :=\sum_{i, l \in \mathbb{Z}} G_{i, l}(f)(x, y) .
\end{aligned}
$$

By Plancherel's theorem we have

$$
\begin{aligned}
\left\|G_{i, l}(f)\right\|_{2}^{2} & =\iint_{\mathbb{R}^{M} \times \mathbb{R}^{N}} \sum_{k, j \in \mathbb{Z}} \int_{d_{\mu}^{j}}^{d_{\mu}^{++1}} \int_{a_{\mu}^{k}}^{a_{\mu}^{k+1}}\left|\omega_{\mu ; r, s}^{\iota, \kappa} *\left(S_{i+k, l+j} S_{i+k, l+j} f\right)(x, y)\right|^{2} \frac{d r d s}{r s} d x d y \\
& =\sum_{k, j \in \mathbb{Z}} \int_{d_{\mu}^{j}}^{a_{\mu}^{j+1}} \int_{a_{\mu}^{k}}^{a_{\mu}^{k+1}} \int_{E_{i+k, l+j}}\left|\widehat{\omega_{\mu ;, r, s}^{\widehat{l}, k}}(\xi, \eta)\right|^{2}|\hat{f}(\xi, \eta)|^{2} d \xi d \eta \frac{d r d s}{r s} \\
& =\sum_{k, j \in \mathbb{Z}} \int_{E_{i+k, l+j}} \int_{a_{\mu}^{j}}^{a_{\mu}^{j+1}} \int_{a_{\mu}^{k}}^{a_{\mu}^{k+1}}\left|\widehat{\omega_{\mu, r, s}^{l, k}}(\xi, \eta)\right|^{2} \frac{d r d s}{r s}|\hat{f}(\xi, \eta)|^{2} d \xi d \eta,
\end{aligned}
$$


where $E_{i+k, l+j}$ is as in (3.6). Without loss of generality, we may assume $\left\{d_{\alpha_{l}}\right\}_{l=1}^{\lambda_{1}}$ and $\left\{v_{\beta_{\kappa}}\right\}_{\kappa=1}^{\lambda_{2}}$ are two sequences of positive numbers. Applying Lemma 2.8, we get

$$
\left\|G_{i, f} f\right\|_{2} \leq C(\mu+1) \tilde{B}_{i, l}\|f\|_{2}
$$

where

$$
\tilde{B}_{i, l}= \begin{cases}B_{\varphi}^{-i d_{\alpha_{l}} / 2} B_{\psi}^{-l v_{\beta_{\kappa}} / 2}, & i, l>-1, \\ B_{\varphi}^{-i d_{\alpha_{l}} / 2} B_{\psi} \epsilon_{2} v_{\beta_{\kappa}} / 2 & i>-1, l \leq-1, \\ B_{\varphi}^{i l_{1} d_{\alpha_{l}} / 2} B_{\psi}^{-l v_{\beta_{\kappa}} / 2}, & i \leq-1, l>-1 \\ B_{\varphi}^{i \epsilon l_{1} d_{\alpha_{l}} / 2} B_{\psi}^{l \epsilon_{2} v_{\beta_{\kappa}} / 2}, & i, l \leq-1\end{cases}
$$

Here $B_{\varphi}, B_{\psi}$ are as in the proof of Theorem 1.1. Next, for $p>2$, let $q=(p / 2)^{\prime}$. Then there exists a function $g \in L^{q}\left(\mathbb{R}^{M} \times \mathbb{R}^{N}\right)$ with $\|g\|_{q} \leq 1$ such that

$$
\begin{aligned}
& \left\|G_{i, l}(f)\right\|_{p}^{2} \\
& =\iint_{\mathbb{R}^{M} \times \mathbb{R}^{N}} \sum_{k, j \in \mathbb{Z}} \int_{a_{\mu}^{j}}^{d_{\mu}^{j+1}} \int_{a_{\mu}^{k}}^{a_{\mu}^{k+1}}\left|\omega_{\mu ; r, s}^{\iota, \kappa} *\left(S_{i+k, l+j} S_{i+k, l+j} f\right)(x, y)\right|^{2} \frac{d r d s}{r s}|g(x, y)| d x d y \\
& \leq \sup _{r, s>0}\left\|\omega_{\mu ; r, s}^{\iota, \kappa}\right\| \\
& \times \sum_{k, j \in \mathbb{Z}} \int_{d_{\mu}^{j}}^{d_{\mu}^{j+1}} \int_{a_{\mu}^{k}}^{a_{\mu}^{k+1}} \iint_{\mathbb{R}^{m} \times \mathbb{R}^{n}}\left|\left(S_{i+k, l+j} S_{i+k, l+j} f\right)(x-u, y-v)\right|^{2} d \omega_{\mu ; r, s}^{\iota, \kappa}(u, v) \frac{d r d s}{r s} \\
& \times|g(x, y)| d x d y \\
& \leq C \iint_{\mathbb{R}^{M} \times \mathbb{R}^{N}} \sum_{k, j \in \mathbb{Z}}\left|S_{i+k, l+j} S_{i+k, l+j} f(x, y)\right|^{2} \mathcal{U}(|g|)(-x,-y) d x d y \\
& \leq C\left\|\left(\sum_{k, j \in \mathbb{Z}}\left|S_{i+k, l+j} S_{i+k, l+j} f\right|^{2}\right)^{1 / 2}\right\|_{p}^{2}\|\mathcal{U}(|g|)\|_{q}
\end{aligned}
$$

where $\mathcal{U}$ is as in Lemma 2.12. By the Littlewood-Paley theory and Lemma 2.12 we have

$$
\left\|G_{i, l}(f)\right\|_{p} \leq C(\mu+1)\|f\|_{p}, \quad p>2 .
$$

Interpolating between (3.13) and (3.15), for any fixed $p>2$, we can choose $\delta_{p} \in(0,1]$ such that

$$
\left\|G_{i, l}(f)\right\|_{p} \leq C(\mu+1) \tilde{B}_{i, l}^{\delta_{p}}\|f\|_{p} .
$$

This, combined with (3.12) and Minkowski's inequality, yields that

$$
\begin{aligned}
\left\|M_{l, \kappa}^{(2)}(f)\right\|_{p} \leq & C\left(\sum_{i, l>-1} B_{\varphi}^{-i d_{\alpha_{l}} \delta_{p} / 2} B_{\psi}^{-l v_{\beta_{\kappa}} \delta_{p} / 2}+\sum_{i>-1, l \leq-1} B_{\varphi}^{-i d_{\alpha_{l}} \delta_{p} / 2} B_{\psi}^{l \epsilon_{2} v_{\beta_{\kappa}} \delta_{p} / 2}\right. \\
& \left.+\sum_{i \leq-1, l>-1} B_{\varphi}^{i \epsilon_{1} d_{\alpha_{l}} \delta_{p} / 2} B_{\psi}^{-l v_{\beta_{\kappa}} \delta_{p} / 2}+\sum_{i, l \leq-1} B_{\varphi}^{i \epsilon_{1} d_{\alpha_{l}} \delta_{p} / 2} B_{\psi}^{l \epsilon_{2} v_{\beta_{\kappa}} \delta_{p} / 2}\right)\|f\|_{p} \\
\leq & C(\mu+1)\|f\|_{p},
\end{aligned}
$$


which, together with (3.11), leads to

$$
\left\|M_{\Omega_{\mu}, \Gamma, \Upsilon}^{(2)}(f)\right\|_{p} \leq C(\mu+1)\|f\|_{p} \quad \text { for } p>2 .
$$

Case $2(\gamma=1)$. For $f \in L^{\infty}\left(\mathbb{R}^{M} \times \mathbb{R}^{N}\right)$ and $h \in U_{1}$, we have

$$
\begin{aligned}
\left|T_{h, \Omega_{\mu}, \Gamma, \Upsilon}(f)(x, y)\right|= & \mid \int_{0}^{\infty} \int_{0}^{\infty} h(r, s) \iint_{S^{m-1} \times S^{n-1}} f\left(x-\Gamma\left(r u^{\prime}\right), y-\Upsilon\left(s v^{\prime}\right)\right) \\
& \times \Omega_{\mu}\left(u^{\prime}, v^{\prime}\right) d \sigma_{m}\left(u^{\prime}\right) d \sigma_{n}\left(v^{\prime}\right) \frac{d r d s}{r s} \mid \\
\leq & C\left\|\Omega_{\mu}\right\|_{L^{1}\left(S^{m-1} \times S^{n-1}\right)}\|h\|_{U_{1}}\|f\|_{\infty} \\
\leq & C\|f\|_{\infty}
\end{aligned}
$$

for every $(x, y) \in \mathbb{R}^{M} \times \mathbb{R}^{N}$. We get from (2.3) that

$$
\left|M_{\Omega_{\mu}, \Gamma, \Upsilon}^{(1)}(f)(x, y)\right|=\sup _{h \in U_{1}}\left|T_{h, \Omega_{\mu}, \Gamma, \Upsilon}(f)(x, y)\right| \leq C\|f\|_{\infty},
$$

which implies

$$
\left\|M_{\Omega_{\mu}, \Gamma, \Upsilon}^{(1)}(f)\right\|_{\infty} \leq C\|f\|_{\infty} .
$$

Case $3(1<\gamma<2)$. For convenience, we set $\digamma(f)=f * \sigma_{\mu ; r, s}^{\lambda_{1}, \lambda_{2}}$. By Cases 1 and 2 we have

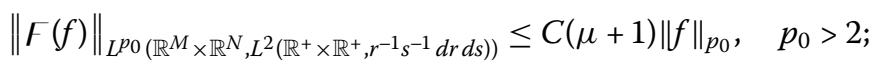

$$
\begin{aligned}
& \|\digamma(f)\|_{L^{\infty}\left(\mathbb{R}^{M} \times \mathbb{R}^{N}, L^{\infty}\left(\mathbb{R}^{+} \times \mathbb{R}^{+}, r^{-1} s^{-1} d r d s\right)\right)} \leq C\|f\|_{\infty} .
\end{aligned}
$$

The real interpolation theorem for Lebesgue mixed norm spaces tells us that

$$
\|\digamma(f)\|_{L^{p}\left(\mathbb{R}^{M} \times \mathbb{R}^{N}, L^{\gamma^{\prime}}\left(\mathbb{R}^{+} \times \mathbb{R}^{+}, r^{-1} s^{-1} d r d s\right)\right)} \leq C(\mu+1)^{2 / \gamma^{\prime}}\|f\|_{p}, \quad \gamma^{\prime}<p<\infty .
$$

This yields (3.10) and completes the proof.

Proof of Theorem 1.2 We shall prove Theorem 1.2 by considering the following two cases.

Case $1(1 \leq \gamma \leq 2)$. We may assume without loss of generality that $\|h\|_{U_{\gamma}}=1$. Then

$$
\left\|T_{h, \Omega, \Gamma, \Upsilon}(f)\right\|_{p} \leq\left\|M_{\Omega, \Gamma, \Upsilon}^{(\gamma)}(f)\right\|_{p} \leq C\|f\|_{p}
$$

Applying Theorem 1.3, we obtain

$$
\left\|T_{h, \Omega, \Gamma, \Upsilon}(f)\right\|_{\infty} \leq C\|f\|_{\infty} \text { for } \gamma=1
$$

and

$$
\left\|T_{h, \Omega, \Gamma, \Upsilon}(f)\right\|_{p} \leq C\|f\|_{p}, \quad \gamma^{\prime} \leq p<\infty, \text { for } 1<\gamma \leq 2,
$$


which, together with a standard duality argument, implies that

$$
\left\|T_{h, \Omega, \Gamma, \Upsilon}(f)\right\|_{p} \leq C\|f\|_{p}, \quad 1<p \leq \gamma, \text { for } 1<\gamma \leq 2 .
$$

Hence, the interpolation theorem tells us that

$$
\left\|T_{h, \Omega, \Gamma, \Upsilon}(f)\right\|_{p} \leq C\|f\|_{p}, \quad 1<p<\infty .
$$

Case $2(\gamma>2)$. By (3.1)-(3.2), (3.9), and Minkowski's inequality we have

$$
\left\|T_{h, \Omega, \Gamma, \Upsilon}(f)\right\|_{p} \leq C \sum_{\mu \in \mathcal{D} \cup\{0\}} \lambda_{\mu}\|h\|_{\mu, \gamma}(\mu+1)^{2 / \gamma^{\prime}}\|f\|_{p} \quad \text { for } 1<p<\infty .
$$

Combining this inequality with (2.5) and the fact that $\|h\|_{\mu, \gamma} \leq\|h\|_{U_{\gamma}}$ implies

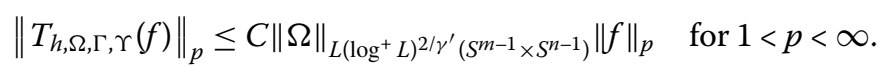

This finishes the proof of Theorem 1.2.

\section{Competing interests}

The authors declare that they have no competing interests.

\section{Authors' contributions}

The authors worked jointly in drafting and approving the final manuscript.

\section{Author details}

${ }^{1}$ College of Mathematics and Systems Science, Shandong University of Science and Technology, Qingdao, Shandong 266590, China. ${ }^{2}$ College of Mathematics and Physics, Fujian University of Technology, Fuzhou, Fujian 350118, China.

${ }^{3}$ School of Mathematics and Statistics, Yili Normal College, Yining, Xinjiang 835000, China.

\section{Acknowledgements}

The authors would link to express their deep gratitude to the referee for his/her carefully reading and invaluable comments. This work was supported by the NNSF of China (No. 11526122), Scientific Research Foundation of Shandong University of Science and Technology for Recruited Talents (No. 2015RCJJ053), Research Award Fund for Outstanding Young Scientists of Shandong Province (No. BS2015SF012), and Support Program for Outstanding Young Scientific and Technological Top-notch Talents of College of Mathematics and Systems Science (No. Sxy2016K01).

Received: 29 March 2016 Accepted: 7 September 2016 Published online: 17 September 2016

\section{References}

1. Fefferman, R: Singular integrals on product domains. Bull. Amer. Math. Soc. 4, 195-201 (1981)

2. Fefferman, R, Stein, EM: Singular integrals on product domains. Adv. Math. 45, 117-143 (1982)

3. Al-Salman, A, Al-Qassem, H, Pan, Y: Singular integrals on product domains. Indiana Univ. Math. J. 55(1), 369-387 (2006)

4. Duoandikoetxea, J: Multiple singular integrals and maximal functions along hypersurfaces. Ann. Inst. Fourier (Grenoble) 36, 185-206 (1986)

5. Liu, F, Zhang, D: Multiple singular integrals and maximal operators with mixed homogeneity along surfaces. Math. Inequal. Appl. 19(2), 499-522 (2016)

6. $\mathrm{Ma}, \mathrm{L}, \mathrm{Fan}, \mathrm{D}, \mathrm{Wu}, \mathrm{H}: \mathrm{L}^{p}$ bounds for singular integrals with rough kernels on product domains. Acta Math. Sin. Engl. Ser. 28, 133-144 (2012)

7. Chen, $J: L^{P}$ boundedness of singular integrals on product domains. Sci. China Ser. A 44(6), 681-689 (2001)

8. Ying, $Y, C$ Chen, $J: L^{p}$ boundedness of a class of singular integrals on product domains. Acta Math. Sin. 46(5), 833-842 (2003)

9. Al-Qassem, $H$, Pan, $Y: L^{p}$ boundedness for singular integrals with rough kernels on product domains. Hokkaido Math. J. 31, 555-613 (2002)

10. Liu, F, Wu, H: Multiple singular integrals and Marcinkiewicz integrals with mixed homogeneity along surfaces. J. Inequal. Appl. 2012, 189 (2012)

11. $\mathrm{Wu}, \mathrm{H}$, Yang, S: On multiple singular integrals along polynomial curves with rough kernels. Acta Math. Sin. Engl. Ser. 24, 177-184 (2008)

12. Al-Qassem, H, Ali, M: Singular integrals related to homogeneous mappings with rough kernels on product domains. Tamkang J. Math. 39(2), 165-176 (2008)

13. Al-Qassem, $H, A$ Ali, $M: L^{p}$ boundedness for singular integral operators with $L\left(\log ^{+} L\right)^{2}$ on product spaces. Kyungpook Math. J. 46, 377-387 (2006) 
14. Al-Salman, A: Parabolic Marcinkiewicz integrals along surfaces on product domains. Acta Math. Sin. Engl. Ser. 27(1), 1-18 (2011)

15. Al-Qassem, H, Pan, Y: A class of maximal operators related to rough singular integrals on product domains. J. Integral Equ. Appl. 17, 331-356 (2005)

16. Cheng, L: Singular integrals related to homogeneous mappings. Mich. Math. J. 47, 407-416 (2000)

17. Fan, D, Guo, K, Pan, Y: $L^{p}$ estimates for singular integrals associated to homogeneous surfaces. J. Reine Angew. Math. $542,1-22(2002)$

18. Liu, F, Mao, S, Wu, H: On rough singular integrals related to homogeneous mappings. Collect. Math. 67(1), 113-132 (2016)

19. Ding, Y: A note on a class of rough maximal operators on product domains. J. Math. Anal. Appl. 232, 222-228 (1999)

20. Al-Salman, A: Maximal operators with rough kernels on product domains. J. Math. Anal. Appl. 311, 338-351 (2005)

21. Fan, D, Pan, Y: Singular integral operators with rough kernels supported by subvarieties. Am. J. Math. 119, 799-839 (1997)

22. Lan, S, Liu, F, Wu, H: Singular integrals and Marcinkiewicz integrals along compound curves on product domains. Adv Math. 43(6), 921-941 (2014)

Submit your manuscript to a SpringerOpen ${ }^{\circ}$ journal and benefit from:

- Convenient online submission

Rigorous peer review

- Immediate publication on acceptance

- Open access: articles freely available online

- High visibility within the field

- Retaining the copyright to your article 